\title{
Hybridized Paracomplete and Paraconsistent Logics
}

\author{
Colin R. Caret \\ Yonsei University
}

\section{Introduction}

In recent years, two non-classical views about negation have gained considerable traction in the philosophical literature 1 The paracomplete view holds that excluded middle (EM) is invalid, while the paraconsistent view holds that explosion (EX) is invalid ${ }^{2}$ Both run contrary to classical logic in distinct but dual ways.

$$
A \therefore B \vee \neg B(\mathrm{EM}) \quad A \wedge \neg A \therefore B(\mathrm{EX})
$$

This paper contributes to the study of paracompleteness and paraconsistency. We present two logics that address the following questions in novel ways. How can the paracomplete theorist characterize the formulas that defy excluded middle while maintaining that not all formulas are of this kind? How can the paraconsistent theorist characterize the formulas that obey explosion while still maintaining that there are some formulas not of this kind?

Our jumping-off point is the observation that (EM) and (EX) are concerned not with negation 'in a vacuum' but the way in which negation interacts with conjunction, in one case, and with disjunction in the other. This is, in part, an artifact of how we have presented these argument forms in order to emphasize their duality in a single-conclusion setting. Explosion is usually presented as an argument form leading from an inconsistent set of premises, $\{A, \neg A\}$, to arbitrary conclusions $B$. In a multiple-conclusion setting we could present excluded middle as the dual argument form leading

\footnotetext{
${ }^{1}$ See, e.g., Dummett (1959), da Costa (1974), Kripke (1975), Priest (2006), Field (2008), Beall (2015).

${ }^{2}$ Throughout the paper, I use capital letters $A, B, C$ as metavariables over formulas and capital letters $X, Y, Z$ for sets of formulas. The displayed argument forms (EM) and (EX) are intended to be read with their premises on the left and their conclusions on the right.
}

Australasian Journal of Logic (14:1) 2017, Article no. 11 
from arbitrary premises, $A$, to a dilemmatic set of conclusions $\{B, \neg B\}$. We will not, however, work with multiple-conclusion logics in this paper, so we opt to present explosion in a slightly unusual way ${ }^{3}$ More generally, throughout the paper we aim to capture, as far as possible, the integral duality between paracomplete and paraconsistent logics. This opens a new avenue for thinking about how logical resources may bear on the expressive questions raised above. We approach the task of characterizing formulas that violate explosion and excluded middle via the treatment of conjunction and disjunction.

The plan of the paper is as follows. For each of our two logics, we give some motivating remarks, articulate its semantics, and compare it to more familiar logics. Much of the paper will then be devoted to proof theory. We explore two kinds of proof theory: axiomatic Hilbert systems and tableaux systems. A proof system of each type is developed and shown to be adequate to its respective target semantics. These systems are largely independent. Either one could be studied without reference to the other, they simply provide an array of tools for working with our two logics. We highlight, in particular, how these logics can describe and reason about their characteristic non-classical phenomena.

\section{Paracomplete Hybrid Logic $\mathrm{MH}$}

\subsection{Semantics and Noteworthy Features}

In the first half of the paper, we explore the propositional logic $\mathrm{MH}$. We use the letter ' $\mathrm{M}$ ' to evoke paracompleteness and ' $\mathrm{N}$ ' to evoke paraconsistency, while the letter ' $\mathrm{H}$ ' indicates a kind of hybridized logic. The logic we define in this section is paracomplete but non-paraconsistent with a hybrid of features found in the well-known logics $\mathrm{K}_{3}$ (Kleene, 1952) and $\mathrm{I}^{2}$ (Sette and Carnielli, 1995), so we call it $\mathrm{MH}$. The matrix semantics for $\mathrm{MH}$ has the same negation operation as $\mathrm{K}_{3}$ and $\mathrm{I}^{2}$, the conjunction of $\mathrm{K}_{3}$, and the implication of $\mathrm{I}^{2}$.

Disjunction, however, has unfamiliar behavior in this logic, so we want to give some brief motivating remarks about its semantics. We take the $\mathrm{K}_{3}$ semantics for negation, conjunction, and disjunction as background and argue for a small modification to its treatment of disjunction. This is premised on the supposition that there are violations of excluded middle.

\footnotetext{
${ }^{3}$ In our paraconsistent logic (and many others) we can exchange any conjunction of premises $\left(A_{1} \wedge \ldots \wedge A_{n}\right)$ with the set of premises $\left\{A_{1}, \ldots, A_{2}\right\}$ salva validitate. This is one reason there should be no harm in our presentation of explosion: it is equivalent to the standard presentation for all intents and purposes.
}

Australasian Journal of Logic (14:1) 2017, Article no. 11 
Suppose, then, that it is rationally permissible to reject some dilemma $(A \vee \neg A)$. That is to say, logic does not, in itself, compel us to accept such a formula. In that case, it is appropriate to call $A$ a locus of rational incompleteness, or more simply an incomplete formula. On most views, incomplete formulas are exceptional, arising from quirks of mind and language such as vagueness or undecidability. Many formulas $B$ are complete in the sense that we ought, instead, to reject $\neg(B \vee \neg B)$ with respect to such formulas. The properties of completeness and incompleteness, so understood, serve a diagnostic semantic role, i.e. incomplete formulas are exactly those that ground the failure of excluded middle. Hence, unlike a formula and its negation - which, by hypothesis, can be jointly rejected - regarding a formula to be either complete or incomplete seems to be categorically required. One posit that easily accommodates all of these assumptions is that $\neg(A \vee \neg A)$ ought to be accepted (in model-theoretic terms, be designated) just when $A$ is incomplete. Given the standard three-valued definition of negation, however, that requires that when $A$ is incomplete, $(A \vee \neg A)$ is false. Moreover, if explosion remains valid, then the $\mathrm{K}_{3}$ semantics for negation, conjunction, and disjunction is almost wholly correct with one exception: a disjunction of incomplete formulas is false. That is the approach of $\mathrm{MH}$.

In less philosophical terms, we can also see this logic as a solution to an engineering problem along the lines of da Costa (1974). Suppose we are interested in a particular articulation of the paracomplete conviction: we want a propositional logic in which excluded middle is invalid but where assertion of this fact is non-contradictory, viz. a premise of the form $\neg(A \vee \neg A)$ does not necessarily entail any arbitrary conclusions whatsoever. It also ought to be straightforward to extend the logic with quantifiers, etc. $\mathrm{MH}$ solves this problem. And while it is not the only possible solution, Beziau and Franceschetto (2015, p.137) point out that the type of semantics we use 'minimizes molecularization (molecular propositions behaving classically)'. So, it has a number of attractive purely formal qualities.

The signature of $\mathrm{MH}$ is below, with a set of formulas FORM defined in the usual way.

$$
\left|p_{i}\right| \neg|\wedge| \vee|\rightarrow|
$$

We also introduce a biconditional in the usual way $(A \leftrightarrow B)={ }_{\text {df. }}(A \rightarrow$ $B) \wedge(B \rightarrow A)$. The set of semantic values of $\mathrm{MH}$ is $\mathbb{S}=\left\{1, \frac{1}{2}, 0\right\}$, the set of designated values is $\mathbb{D}=\{1\}$, and an $\mathrm{MH}$ valuation is any function $v: F O R M \rightarrow \mathbb{S}$ closed under the following operations. 


\begin{tabular}{|c|c|}
\hline$\neg$ & \\
\hline 1 & 0 \\
$\frac{1}{2}$ & $\frac{1}{2}$ \\
0 & 1 \\
\hline
\end{tabular}

\begin{tabular}{|c|ccc|}
\hline$\wedge$ & 1 & $\frac{1}{2}$ & 0 \\
\hline 1 & 1 & $\frac{1}{2}$ & 0 \\
$\frac{1}{2}$ & $\frac{1}{2}$ & $\frac{1}{2}$ & 0 \\
0 & 0 & 0 & 0 \\
\hline
\end{tabular}

\begin{tabular}{|c|ccc|}
\hline$\vee$ & 1 & $\frac{1}{2}$ & 0 \\
\hline 1 & 1 & 1 & 1 \\
$\frac{1}{2}$ & 1 & 0 & $\frac{1}{2}$ \\
0 & 1 & $\frac{1}{2}$ & 0 \\
\hline
\end{tabular}

\begin{tabular}{|c|ccc|}
\hline$\rightarrow$ & 1 & $\frac{1}{2}$ & 0 \\
\hline 1 & 1 & 0 & 0 \\
$\frac{1}{2}$ & 1 & 1 & 1 \\
0 & 1 & 1 & 1 \\
\hline
\end{tabular}

We define the consequence relation of $\mathrm{MH}$ as follows.

$X \vDash_{\mathrm{MH}} C$ iff on all $\mathrm{MH}$ valuations $v$, either $v(A) \notin \mathbb{D}$ for some $A \in X$ or $v(C) \in \mathbb{D}$

Before delving into proof-theoretic matters, we highlight some features of potential interest in this logic. $\mathrm{MH}$ has a paracomplete but nonparaconsistent negation as exemplified by the failure of excluded middle (a) and the presence of explosion (b). It also has asymmetrically defined conjunction and disjunction connectives for which the customary inferences (c)-(g) hold, but for which only some of the DeMorgan laws hold (h) while others fail (i). The conditional satisfies conditional proof (j) and classical logical equivalents are intersubstitutible $(\mathrm{k})$.

(a) $A \not \nvdash_{\mathrm{MH}} B \vee \neg B$

(b) $A \wedge \neg A \vDash_{\mathrm{MH}} B$

(c) $A \wedge B \vDash_{\mathrm{MH}} A$

(d) $A \vDash_{\mathrm{MH}} B$ and $A \vDash_{\mathrm{MH}} C \Longrightarrow A \vDash_{\mathrm{MH}} B \wedge C$

(e) $A \vDash_{\mathrm{MH}} A \vee B$

(f) $A \vDash_{\mathrm{MH}} C$ and $B \vDash_{\mathrm{MH}} C \Longrightarrow A \vee B \vDash_{\mathrm{MH}} C$

(g) $A \wedge(B \vee C) \vDash_{\mathrm{MH}}(A \wedge B) \vee(A \wedge C)$

(h) $\neg(A \wedge B) \vDash_{\mathrm{MH}} \neg A \vee \neg B$

(i) $\neg(A \vee B) \not \nvdash_{\mathrm{MH}} \neg A \wedge \neg B$

(j) $X, A \vDash_{\mathrm{MH}} B \Longrightarrow X \vDash_{\mathrm{MH}} A \rightarrow B$

(k) $A \leftrightarrow B, \neg A \leftrightarrow \neg B \vDash_{\mathrm{MH}} \Psi(A) \leftrightarrow \Psi(B)$ where $\Psi(B)$ is obtained from $\Psi(A)$ by replacing zero or more instances of $A$ with $B$. 
The failure of (i) is symptomatic of the fact that, in virtue of its disjunction operation, $\mathrm{MH}$ has the resources to express when a formula is incomplete as follows: $v(\neg(A \vee \neg A)) \in \mathbb{D}$ iff $v(A)=\frac{1}{2}$. In many paracomplete but non-paraconsistent logics, denying an instance of excluded middle entails a contradiction, which is trivializing. This results from the DeMorgan law that fails to hold in $\mathrm{MH}$. Denying an instance of excluded middle is, thus, non-trivial in $\mathrm{MH}$ because doing so carries no contradictory implications. As a result, we can define an incompleteness operator $\nabla A={ }_{\mathrm{df}} \neg(A \vee \neg A)$ that has the following features in $\mathrm{MH}$.

(j) $A \vDash_{\mathrm{MH}}(B \vee \neg B) \vee \nabla B$

(k) $A \leftrightarrow \neg A \vDash_{\mathrm{MH}} \nabla A$

(l) $\nabla A \wedge \nabla B \vDash_{\mathrm{MH}} \nabla(A \wedge B)$

$(\mathbf{m}) \nabla(A \vee B) \vDash_{\mathrm{MH}} \nabla A \vee \nabla B$

(n) $\neg(A \vee B) \vDash_{\mathrm{MH}}(\neg A \wedge \neg B) \vee \nabla(A \wedge B)$

(o) $A, \nabla B \vDash_{\mathrm{MH}} \neg(A \rightarrow B)$

(p) $\nabla A,(A \rightarrow B) \not \nvdash_{\mathrm{MH}} \neg B \rightarrow \neg A$

If we think of $\nabla$ as expressing rational incompleteness, i.e. that one should neither assert nor deny such formulas, then most of these laws are intuitively correct. We have a law of 'excluded thirds' that says that any formula can either be asserted, denied, or neither of these because it is a locus of incompleteness (j). We get a near-cousin of our missing DeMorgan law (n), which arises from the formal strategy of $\mathrm{MH}$, viz. to extract expressive power from a novel treatment of disjunction. The formulas with respect to which a theory is incomplete are those that are equivalent to their own negations (k) and incompleteness restricts contraposition (o)-(p).

Our conditional generalizes material implication, insofar as a conditional formula is true just when its antecedent is undesignated or its consequent is designated. As a result, $\mathrm{MH}$ has strong implicational laws, tautologies, and vacuous entailments like $A \vDash_{\mathrm{MH}} B \rightarrow B$, all of which fail in the strong Kleene logic $\mathrm{K}_{3}$. It has the rule of absorption $A \rightarrow(A \rightarrow B) \vDash_{\mathrm{MH}} A \rightarrow B$ which fails even in the three-valued Lukasiewicz logic $\bigsqcup_{3}$. So it is a strong paracomplete logic. On the other hand, there are other inferences that fail in $\mathrm{MH}$, such as $(A \rightarrow B) \rightarrow B \nvdash_{\mathrm{MH}} A \vee B$, but that hold in Eukasiewicz logic. Although $\mathrm{MH}$ and $\iota_{3}$ each strictly extends Kleene's logic, neither is 
properly contained in the other. $\mathrm{MH}$ also, notably, retains classical metainferences such as proof by cases (f), which fail in supervaluational logics with an 'indeterminacy' operator 4 Having thus situated $\mathrm{MH}$ in the logical terrain, we turn to its proof theory.

\subsection{Hilbert System HMH}

In this subsection, we develop a Hilbert system $\mathrm{HMH}$ and show that it is sound and complete for our paracomplete hybrid logic $\mathrm{MH}$. Any uniform substitution of formulas into metavariable positions in one of the following schemas is an axiom of $\mathrm{HMH}$.

(Ax.1) $A \rightarrow(B \rightarrow A)$

(Ax.2) $(A \rightarrow(B \rightarrow C)) \rightarrow((A \rightarrow B) \rightarrow(A \rightarrow C))$

(Ax.3) $(A \wedge B) \rightarrow A$

(Ax.4) $(A \wedge B) \rightarrow B$

(Ax.5) $(A \rightarrow B) \rightarrow((A \rightarrow C) \rightarrow(A \rightarrow(B \wedge C)))$

(Ax.6) $A \rightarrow(A \vee B)$

(Ax.7) $B \rightarrow(A \vee B)$

(Ax.8) $(A \rightarrow C) \rightarrow((B \rightarrow C) \rightarrow((A \vee B) \rightarrow C))$

(Ax.9) $\neg \neg A \leftrightarrow A$

(Ax.10) $(A \vee \neg A) \vee \nabla A$

(Ax.11) $(A \rightarrow B) \vee \neg(A \rightarrow B)$

(Ax.12) $(A \vee \neg A) \rightarrow((A \rightarrow B) \rightarrow(\neg B \rightarrow \neg A))$

(Ax.13) $\neg(A \wedge B) \leftrightarrow(\neg A \vee \neg B)$

(Ax.14) $\neg(A \vee B) \leftrightarrow((\neg A \wedge \neg B) \vee(\nabla A \wedge \nabla B))$

(Ax.15) $(\neg A \vee \nabla A) \rightarrow(A \rightarrow B)$

(Ax.16) $(A \wedge(\neg B \vee \nabla B)) \rightarrow \neg(A \rightarrow B)$

\footnotetext{
${ }^{4}$ See Williamson (1994, p.152).
} 
We also have a rule of modus ponens.

$$
\text { (MP) } A \rightarrow B, A \Longrightarrow B
$$

A derivation in $\mathrm{HMH}$ from the set of assumptions $X$ to conclusion $C$ is a finite sequence of formulas $A_{1}, \ldots, A_{n}$ such that $A_{n}=C$ and such that every $A_{i}$ in the sequence is either a member of $X$, an axiom of $\mathrm{HMH}$, or follows from previous formulas in the sequence by MP. We write the following to express that such a derivation exists.

$$
X \vdash_{\mathrm{HMH}} C
$$

A formula that is derivable from the empty set is a theorem of $\mathrm{HMH}$.

$\mathrm{HMH}$ has a deduction theorem because it contains the full axioms of intuitionistic implication. It also has a law reflecting the fact that 'incompleteness' is fixed over negation. We note two different explosion theorems and other useful derived rules below. These results are established in the appendix.

(Iden) $A \rightarrow A$

(IFN) $\nabla A \leftrightarrow \nabla \neg A$

(MEX) $\neg A \rightarrow(A \rightarrow B)$ and $\nabla A \rightarrow(\neg A \rightarrow B)$

(Adj) $A, B \Longrightarrow A \wedge B$

(Tran) $A \rightarrow B, B \rightarrow C \Longrightarrow A \rightarrow C$

(Perm) $A \rightarrow(B \rightarrow C) \Longrightarrow B \rightarrow(A \rightarrow C)$

(MRed) $A \rightarrow(\neg A \vee \nabla A) \Longrightarrow \neg A \vee \nabla A$

(IncC) $\nabla(A \wedge B) \Longrightarrow(A \vee \nabla A)$ and $(B \vee \nabla B)$

(IncD) $\nabla(A \vee B) \Longrightarrow(\neg A \vee \nabla A)$ and $(\neg B \vee \nabla B)$

Following the example of Goldberg et al. (1974), we give a Henkin-style completeness proof for $\mathrm{HMH}$ with respect to $\mathrm{MH}$. We need a few definitions and lemmas.

- A set of formulas $X$ is $\mathrm{HMH}$-trivial iff $X \vdash_{\mathrm{HMH}} A$ for all $A$; it is $\mathrm{HMH}$-non-trivial otherwise. 
- A set of formulas $X$ is maximal $\mathrm{HMH}$-non-trivial iff it is $\mathrm{HMH}$-nontrivial and $X \cup\{B\}$ is $\mathrm{HMH}$-trivial for all formulas $B \notin X$.

- An $\mathrm{HMH}$-filter is a set of formulas that contains all $\mathrm{MH}$ axioms and is closed under MP.

Lemma 2.2.1. If $X$ is maximal $\mathrm{HMH}$-non-trivial, then $X$ is an $\mathrm{HMH}$-filter.

Proof. Fix any maximal $\mathrm{HMH}$-non-trivial set $X$. Let $A$ be either an $\mathrm{MH}$ axiom or a formula such that there are $(B \rightarrow A) \in X$ and $B \in X$. In both cases $X \vdash_{\mathrm{HMH}} A$. Suppose, however, that $A \notin X$. Then by maximality, $X \cup\{A\}$ is $\mathrm{HMH}$-trivial, hence $X, A \vdash_{\mathrm{HMH}} C$ for all $C$. In that case, by the deduction theorem, $X \vdash_{\mathrm{HMH}} A \rightarrow C$ for all $C$ and so, by MP, we have $X \vdash_{\mathrm{HMH}} C$ for all $C$. But this contradicts the non-triviality of $X$. Thus, $A \in X$.

Clearly, a maximal HMH-non-trivial set contains all theorems and is closed under derivability. For example, such sets are closed under adjunction and transitivity. We also show that such sets are prime, which will be useful to know in what follows.

Corollary (Primeness). If $X$ is maximal $\mathrm{HMH}$-non-trivial, then $X$ is prime; in particular, with respect to any formula $C$, exactly one of $C \in X, \neg C \in X$, or $\nabla C \in X$.

Proof. Fix any maximal HMH-non-trivial set $X$ with $(A \vee B) \in X$. Suppose, however, that $A \notin X$ and $B \notin X$. Then by maximality, both $X \cup\{A\}$ and $X \cup\{B\}$ are $\mathrm{HMH}$-trivial. So, by $\mathrm{RBC}$, we have $X, A \vee B \vdash_{\mathrm{HMH}} C$ for all $C$. But since $(A \vee B) \in X$ already it follows that $X \vdash_{\mathrm{HMH}} C$ for all $C$, contradicting the non-triviality of $X$. Thus, either $A \in X$ or $B \in X$. In particular, if $X$ is any maximal $\mathrm{HMH}$-non-trivial set, then with respect to any formula $C$, at least one of $C \in X, \neg C \in X$, or $\nabla C \in X$ by Ax.10 and Lemma 2.2.1, and at most one of $C \in X, \neg C \in X$, or $\nabla C \in X$ by the explosion theorems MEX.

The next three lemmas will help simplify the rest of the presentation.

Lemma 2.2.2. If $X$ is maximal $\mathrm{HMH}$-non-trivial and $\nabla(A \wedge B) \in X$, then either we have both $\nabla A, \nabla B \in X$ or both $\nabla A, B \in X$ or both $A, \nabla B \in X$.

Proof. Fix maximal HMH-non-trivial set $X$ with $\nabla(A \wedge B) \in X$. Then both $(A \vee \nabla A) \in X$ and $(B \vee \nabla B) \in X$ by Lemma 2.2.1 and IncC. So, 
either $A \in X$ or $\nabla A \in X$ by primeness, and either $B \in X$ or $\nabla B \in X$ by primeness. This gives us four possibilities: either we have both $A, B \in X$ or both $\nabla A, \nabla B \in X$ or both $\nabla A, B \in X$ or both $A, \nabla B \in X$. But the first case is not really possible as it would trivialize $X$ by adjunction, MEX, and Perm.

Lemma 2.2.3. If $X$ is maximal $\mathrm{HMH}$-non-trivial and $\nabla(A \vee B) \in X$, then either we have both $\nabla A, \neg B \in X$ or both $\neg A, \nabla B \in X$.

Proof. Fix maximal $\mathrm{HMH}$-non-trivial set $X$ with $\nabla(A \vee B) \in X$. Then both $(\neg A \vee \nabla A) \in X$ and $(\neg B \vee \nabla B) \in X$ by Lemma 2.2.1 and IncD. So, either $\neg A \in X$ or $\nabla A \in X$ by primeness, and either $\neg B \in X$ or $\nabla B \in X$ by primeness. This gives us four possibilities: either we have both $\neg A, \neg B \in X$ or both $\nabla A, \nabla B \in X$ or both $\nabla A, \neg B \in X$ or both $\neg A, \nabla B \in X$. But the first two cases are not really possible. If both $\neg A, \neg B \in X$, then $(\neg A \wedge \neg B) \in X$ by adjunction, hence $((\neg A \wedge \neg B) \vee(\nabla A \wedge \nabla B)) \in X$ by Ax.6. If both $\nabla A, \nabla B \in X$, then $(\nabla A \wedge \nabla B) \in X$ by adjunction, hence $((\neg A \wedge \neg B) \vee(\nabla A \wedge \nabla B)) \in X$ by Ax.7. In either case, $\neg(A \vee B) \in X$ by Ax.14, which would trivialize $X$ by adjunction and MEX.

Lemma 2.2.4. If $X$ is maximal $\mathrm{HMH}$-non-trivial and either $\neg(\neg A \vee \nabla A) \in$ $X$ or $\nabla(\neg A \vee \nabla A) \in X$, then $A \in X$.

Proof. Fix maximal HMH-non-trivial set $X$ with $\neg(\neg A \vee \nabla A) \in X$. Then $(\neg \neg A \wedge \neg \nabla A) \in X$ or $(\nabla \neg A \wedge \nabla \nabla A) \in X$ by Ax.14 and primeness. In the first case, $A \in X$ by simplification and Ax.9. The second case is not really possible as it would trivialize $X$ by IFN and MEX. A similar result holds if we start with $\nabla(\neg A \vee \nabla A) \in X$ and apply IncD and MEX.

We can now establish the following important relationship.

Lemma 2.2.5. If $X$ is $\mathrm{HMH}$-non-trivial, then $X$ is satisfied on some $\mathrm{MH}$ valuation.

Proof. Fix any $\mathrm{HMH}$-non-trivial set of formulas $X$. We want to extend this set to a maximal $\mathrm{HMH}$-non-trivial superset $X^{*}$. Let $A_{1}, \ldots, A_{n}$ be an enumeration of FORM and define $X^{*}$ recursively as follows.

$$
\begin{gathered}
X_{0}=X \\
X_{n+1}= \begin{cases}X_{n} \cup\left\{A_{n}\right\} & \text { if this is HMH-non-trivial } \\
X_{n} & \text { otherwise }\end{cases}
\end{gathered}
$$




$$
X^{*}=\bigcup_{n \in \mathbb{N}} X_{n}
$$

Succession preserves non-triviality by design, so each of the $X_{i}$ for $i \in \mathbb{N}$ is $\mathrm{HMH}$-non-trivial. In that case, the limit $X^{*}$ must be $\mathrm{HMH}$-non-trivial as well. Otherwise we would have $X^{*} \vdash_{\mathrm{HMH}} p \wedge \neg p$ and, since derivations are finite, that would require $X_{i} \vdash_{\mathrm{HMH}} p \wedge \neg p$ for some $X_{i}$, in which case that $X_{i}$ would be trivial by MEX.

Fix an $\mathrm{MH}$ valuation $v_{X^{*}}$ defined as follows for each atomic formula $p$. By the established primeness of any maximal $\mathrm{HMH}$-non-trivial set, this is a well-defined valuation.

$$
v_{X^{*}}(p)=\left\{\begin{array}{ccc}
1 & \text { if } & p \in X^{*} \\
0 & \text { if } & \neg p \in X^{*} \\
\frac{1}{2} & \text { if } & \nabla p \in X^{*}
\end{array}\right.
$$

We show that this valuation extends to all formulas $A$ as follows.

$$
v_{X^{*}}(A)=\left\{\begin{array}{lll}
1 & \text { if } & A \in X^{*} \\
0 & \text { if } & \neg A \in X^{*} \\
\frac{1}{2} & \text { if } & \nabla A \in X^{*}
\end{array}\right.
$$

(I) Base case: holds by definition.

(II) First inductive case: $A$ is a formula of the form $B \wedge C$.

(a) Suppose that $A \in X^{*}$, i.e. that $(B \wedge C) \in X^{*}$. By simplification $B \in X^{*}$ and $C \in X^{*}$. Then $v_{X^{*}}(B)=v_{X^{*}}(C)=1$ by IH, and so $v_{X^{*}}(B \wedge C)=v_{X^{*}}(A)=1$.

(b) Suppose that $\neg A \in X^{*}$, i.e. that $\neg(B \wedge C) \in X^{*}$. Then by Ax.14 and closure $(\neg B \vee \neg C) \in X^{*}$. By primeness $\neg B \in X^{*}$ or $\neg C \in X^{*}$. If $\neg B \in X^{*}$, then $v_{X^{*}}(B)=0$ by IH. If $\neg C \in X^{*}$, then $v_{X^{*}}(C)=0$ by IH. Thus, in every case, $v_{X^{*}}(B \wedge C)=v_{X^{*}}(A)=0$.

(c) Suppose that $\nabla A \in X^{*}$, i.e. that $\nabla(B \wedge C) \in X^{*}$. Then either we have both $\nabla B, \nabla C \in X^{*}$ or both $\nabla B, C \in X^{*}$ or both $B, \nabla C \in X^{*}$ by Lemma 2.2.2. If both $\nabla B, \nabla C \in X^{*}$, then $v_{X^{*}}(B)=v_{X^{*}}(C)=\frac{1}{2}$ by IH. If both $\nabla B, C \in X^{*}$, then $v_{X^{*}}(B)=\frac{1}{2}$ and $v_{X^{*}}(C)=1$ by IH. If both $B, \nabla C \in X^{*}$, then $v_{X^{*}}(B)=1$ and $v_{X^{*}}(C)=\frac{1}{2}$ by IH. Thus, in every case, $v_{X^{*}}(B \wedge C)=v_{X^{*}}(A)=\frac{1}{2}$. 
(III) Second inductive case: $A$ is a formula of the form $B \vee C$.

(a) Suppose that $A \in X^{*}$, i.e. that $(B \vee C) \in X^{*}$. By primeness $B \in X^{*}$ or $C \in X^{*}$. If $B \in X^{*}$, then $v_{X^{*}}(B)=1$ by IH. If $C \in X^{*}$, then $v_{X^{*}}(C)=1$ by IH. Thus, in every case, $v_{X^{*}}(B \vee C)=v_{X^{*}}(A)=1$.

(b) Suppose that $\neg A \in X^{*}$, i.e. that $\neg(B \vee C) \in X^{*}$. Then by Ax.14 and closure $((\neg B \wedge \neg C) \vee(\nabla B \wedge \nabla C)) \in X^{*}$. By primeness and simplification either we have both $\neg B, \neg C \in X^{*}$ or both $\nabla B, \nabla C \in X^{*}$. If both $\neg B, \neg C \in X^{*}$, then $v_{X^{*}}(B)=v_{X^{*}}(C)=$ 0 by IH. If both $\nabla B, \nabla C \in X^{*}$, then $v_{X^{*}}(B)=v_{X^{*}}(C)=\frac{1}{2}$ by IH. Thus, in every case, $v_{X^{*}}(B \vee C)=v_{X^{*}}(A)=0$.

(c) Suppose that $\nabla A \in X^{*}$, i.e. that $\nabla(B \vee C) \in X^{*}$. Then either we have both $\nabla B, \neg C \in X^{*}$ or both $\neg B, \nabla C \in X^{*}$ by Lemma 2.2.3. If both $\nabla B, \neg C \in X^{*}$, then $v_{X^{*}}(B)=\frac{1}{2}$ and $v_{X^{*}}(C)=0$ by IH. If both $\neg B, \nabla C \in X^{*}$, then $v_{X^{*}}(B)=0$ and $v_{X^{*}}(C)=\frac{1}{2}$ by IH. Thus, in every case, $v_{X^{*}}(B \vee C)=v_{X^{*}}(A)=\frac{1}{2}$.

(IV) Third inductive case: $A$ is a formula of the form $B \rightarrow C$.

(a) Suppose that $A \in X^{*}$, i.e. that $(B \rightarrow C) \in X^{*}$. Then by MEX, Perm, Ax.16, Tran, and MRed we have $(\neg(B \wedge(\neg C \vee$ $\nabla C)) \vee \nabla(B \wedge(\neg C \vee \nabla C))) \in X^{*}$ and so either (1) $\neg(B \wedge$ $(\neg C \vee \nabla C)) \in X^{*}$ or $(2) \nabla(B \wedge(\neg C \vee \nabla C)) \in X^{*}$. If (1) holds, then either $\neg B \in X^{*}$ or $\neg(\neg C \vee \nabla C) \in X^{*}$ by Ax.13 and primeness. In the first case, $v_{X^{*}}(B)=0$ by IH. In the second case, $(\neg \neg C \wedge \neg \nabla C) \in X^{*}$ or $(\nabla \neg C \wedge \nabla \nabla C) \in X^{*}$ by Ax.14 and primeness, but the latter trivializes $X^{*}$; thus, in fact, we have $\neg \neg C \in X^{*}$ and so $C \in X^{*}$ and so $v_{X^{*}}(C)=1$ by IH. If (2) holds, then by Lemma 2.2 .2 we have both $\nabla B, \nabla(\neg C \vee \nabla C) \in X^{*}$ or both $\nabla B,(\neg C \vee \nabla C) \in X^{*}$ or both $B, \nabla(\neg C \vee \nabla C) \in X^{*}$. In the first and second cases, $v_{X^{*}}(B)=\frac{1}{2}$ by IH. In the third case, $C \in X^{*}$ by MP and so $v_{X^{*}}(C)=1$ by IH. Thus, in every case, $v_{X^{*}}(B \rightarrow C)=v_{X^{*}}(A)=1$.

(b) Suppose that $\neg A \in X^{*}$, i.e. that $\neg(B \rightarrow C) \in X^{*}$. Then by MEX, Ax.15, Tran, and MRed we have $(\neg((\neg B \vee \nabla B) \vee C) \vee \nabla((\neg B \vee$ $\nabla B) \vee C)) \in X^{*}$ and so either $(1) \neg((\neg B \vee \nabla B) \vee C) \in X^{*}$ or (2) $\nabla((\neg B \vee \nabla B) \vee C) \in X^{*}$. If (1) holds, then either $(\neg(\neg B \vee \nabla B) \wedge \neg C) \in X^{*}$ or $(\nabla(\neg B \vee \nabla B) \wedge \nabla C) \in X^{*}$ by Ax.14 
and primeness. In the first case, $B \in X^{*}$ by Lemma 2.2 .4 and $\neg C \in X^{*}$, so $v_{X^{*}}(B)=1$ and $v_{X^{*}}(C)=0$ by IH. In the second case, $B \in X^{*}$ by Lemma 2.2 .4 and $\nabla C \in X^{*}$, so $v_{X^{*}}(B)=1$ and $v_{X^{*}}(C)=\frac{1}{2}$ by IH. If (2) holds, then by Lemma 2.2 .3 we have both $\nabla(\neg B \vee \nabla B), \neg C \in X^{*}$ or both $\neg(\neg B \vee \nabla B), \nabla C \in X^{*}$. In both cases, $B \in X^{*}$ by Lemma 2.2 .4 and we either have $\neg C \in X^{*}$ or $\nabla C \in X^{*}$ and so $v_{X^{*}}(B) \in \mathbb{D}$ and $v_{X^{*}}(C) \notin \mathbb{D}$ by IH. Thus, in every case, $v_{X^{*}}(B \rightarrow C)=v_{X^{*}}(A)=0$.

(c) The third option, $\nabla A \in X^{*}$, i.e. $\nabla(B \rightarrow C) \in X^{*}$, is not really possible. For if it holds, then by MEX and Perm we have $((B \rightarrow C) \rightarrow D) \in X^{*}$ for all $D$, by MEX we have $(\neg(B \rightarrow C) \rightarrow$ $D) \in X^{*}$ for all $D$, hence $(((B \rightarrow C) \vee \neg(B \rightarrow C)) \rightarrow D) \in X^{*}$ by Ax.8. Then $D \in X^{*}$ for all $D$ by Ax.11, contradicting the non-triviality of $X^{*}$.

(V) Fourth inductive case: $A$ is a formula of the form $\neg B$. In case $B$ is atomic or it has conjunction, disjunction, or implication as its main connective, this is covered by steps (II)b. (III)b and (IV)b. The remaining case is where $B$ itself is of the form $\neg C$.

(a) Suppose that $A \in X^{*}$, i.e. that $\neg \neg C \in X^{*}$. Then by Ax.9 and closure $C \in X^{*}$ and so, by IH, $v_{X^{*}}(C)=v_{X^{*}}(\neg \neg C)=v_{X^{*}}(A)=$ 1 .

(b) Suppose that $\neg A \in X^{*}$, i.e. that $\neg \neg \neg C \in X^{*}$. Then by Ax.9 and closure $\neg C \in X^{*}$ and so, by $\mathrm{IH}, v_{X^{*}}(C)=v_{X^{*}}(\neg \neg C)=$ $v_{X^{*}}(A)=0$.

(c) Suppose that $\nabla B \in X^{*}$, i.e. that $\nabla \neg \neg C \in X^{*}$. Then by IFN and closure $\nabla C \in X^{*}$ and so, by IH, $v_{X^{*}}(C)=v_{X^{*}}(\neg \neg C)=$ $v_{X^{*}}(A)=\frac{1}{2}$.

It follows that $A \in X^{*}$ iff $v_{X^{*}}(A) \in \mathbb{D}$ for all formulas $A$. This shows that $X^{*}$ and any of its subsets, including our original set $X$, are satisfied on some $\mathrm{MH}$ valuation.

This is all that we need to establish completeness for our Hilbert system.

Theorem 2.2.6 (Completeness of $\mathrm{HMH}$ ). If $X \vDash_{\mathrm{MH}} A$, then $X \vdash_{\mathrm{HMH}} A$.

Proof. Suppose that $X \vDash_{\mathrm{MH}} A$. Then there is no $\mathrm{MH}$ valuation on which $v(B) \in \mathbb{D}$ for all $B \in X$ and $v(A) \notin \mathbb{D}$. In that case, $X \cup\{\neg A \vee \nabla A\}$ is unsatisfied on all $\mathrm{MH}$ valuations. It follows by Lemma 2.2.5 that 
$X \cup\{\neg A \vee \nabla A\}$ is $\mathrm{HMH}$-trivial. Then $X, \neg A \vee \nabla A \vdash_{\mathrm{HMH}} A$ and so we have $X \vdash_{\mathrm{HMH}} \neg A \rightarrow A$ and $X \vdash_{\mathrm{HMH}} \nabla A \rightarrow A$ by RBC and the deduction theorem. We also have $X \vdash_{\mathrm{HMH}} A \rightarrow A$ by the Iden theorem, hence $X \vdash_{\mathrm{HMH}}((A \vee \neg A) \vee \nabla A) \rightarrow A$ by Ax.8 and MP. Therefore, by Ax.10 and MP we have $X \vdash_{\mathrm{HMH}} A$.

Theorem 2.2.7 (Soundness of $\mathrm{HMH}$ ). If $X \vdash_{\mathrm{HMH}} A$, then $X \vDash_{\mathrm{MH}} A$.

Proof Sketch. This result is more straightforward. Let $X \vdash_{\mathrm{HMH}} A$ and fix any $\mathrm{HMH}$ derivation from $X$ to $A$ that witnesses the fact. By induction on the length of derivations, we can show that if an $\mathrm{MH}$ valuation $v$ assigns $v(B)=1$ for all $B \in X$, then $v(A)=1$. For the base case, $A$ can only be an $\mathrm{HMH}$ axiom. It is straightforward if tedious to show that every axiom is an $\mathrm{MH}$ tautology. For the inductive case, we only need the fact that if $v(C)=1$ and $v(C \rightarrow D)=1$ then $v(D)=1$, i.e. that MP preserves designation.

This demonstrates the adequacy of $\mathrm{HMH}$ with respect to $\mathrm{MH}$. Since derivations in $\mathrm{HMH}$ are finite, it follows quickly that consequence in $\mathrm{MH}$ is compact. This will be helpful in the next section.

Corollary (Compactness of MH). $X \vDash_{\mathrm{MH}} A$ iff $X^{\prime} \vDash_{\mathrm{MH}} A$ for some finite $X^{\prime} \subseteq X$.

Hilbert systems, of course, have their limitations. Derivations are clunky and not always apparent. For that reason, we now go on to develop a tableaux system as well.

\subsection{Tableaux System TMH}

In this subsection, we develop a tableaux system $\mathrm{TMH}$ and show that it, too, is sound and complete for $\mathrm{MH}$. Our tableaux are downward-branching trees of signed formulas. The metalanguage includes the signs $\oplus$ and $\ominus$, informally representing the semantic status of having a designated or undesignated value.

Given a finite premise set $X$ and conclusion $C$, the root of the TMH tree from $X$ to $C$ is a list consisting of $B \oplus$ for all $B \in X$ and $C \ominus$. Starting from this initial list, we apply the expansion rules below to construct the tree. The expansion rules are sorted by the sign and main connective of the signed formulas to which they may be applied. At each stage of the construction, a signed formula is resolved if the appropriate expansion rule has been applied to it; otherwise it is unresolved. In pen-and-paper exercises, one can keep track of this by marking resolved formulas with, e.g., a check 
mark. Each stage of the construction proceeds by selecting an unresolved signed formula $A \oplus / \ominus$, then listing the signed formulas generated by the appropriate expansion rule at all end-points of the tree below $A \oplus / \ominus$ 's node.
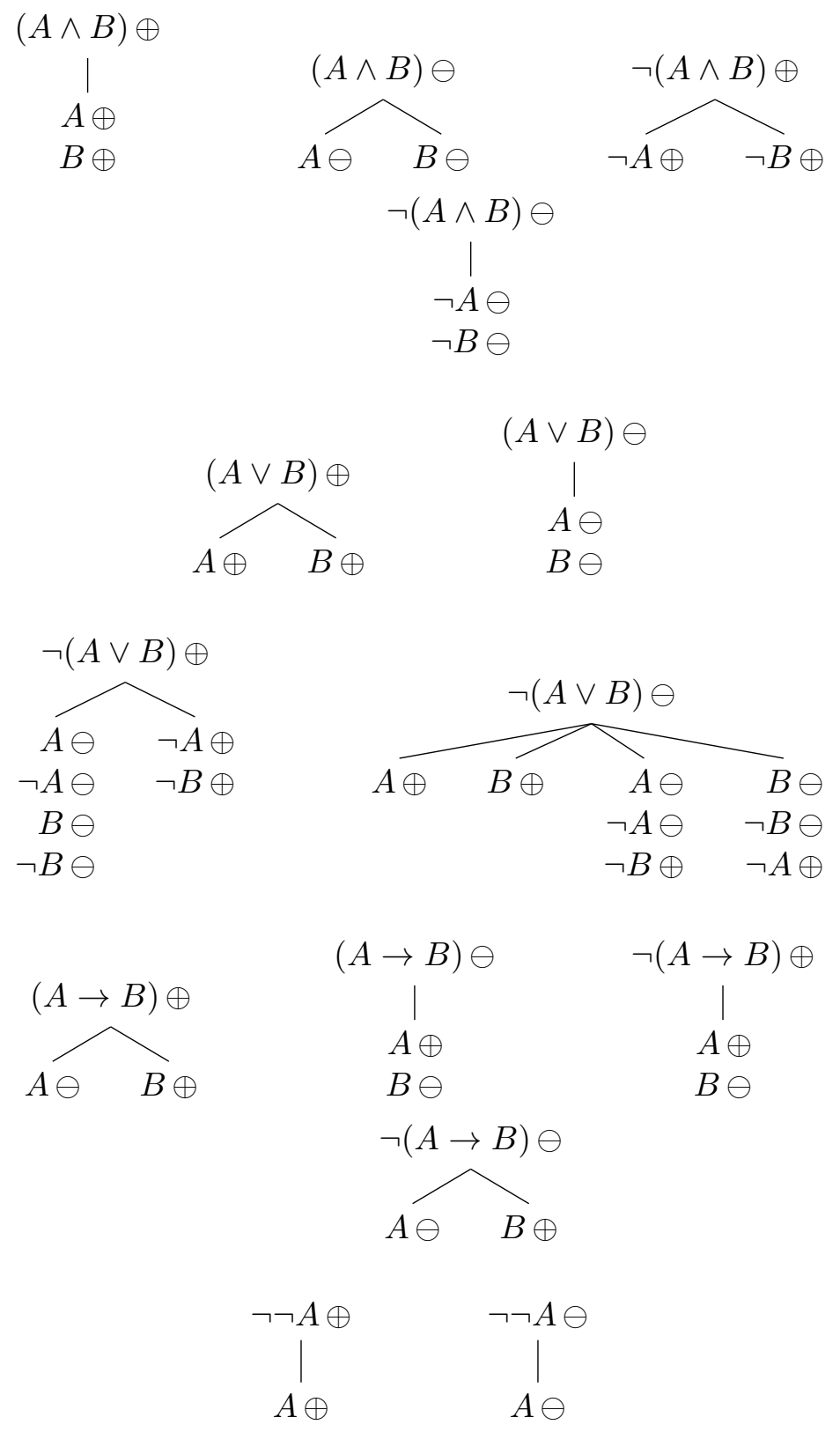

Australasian Journal of Logic (14:1) 2017, Article no. 11 
A branch of a TMH tree is any path from an end-point back up the root. We can think of an expanding branch of the tree as a search for an $\mathrm{MH}$ counter-model to the original argument. At any stage of the construction, a branch is closed if, for any formula $A$, both $A \oplus$ and $A \ominus$ occur on that branch or both $A \oplus$ and $\neg A \oplus$ occur on that branch. Such a branch has failed to produce a viable counter-model. When every rule that can be applied has been applied, the tree is finished. Every rule generates a finite number of extensions of any branch where it is applied. The formulas generated by an expansion rule are always syntactically simpler than the formula at the node where it was applied. So, regardless of how complex the premises of an argument, it will always have a finite, finished TMH tree.

If every branch of a finished tree is closed, the tree is closed; otherwise it is open. The argument from arbitrary premise set $X$ to conclusion $C$ is provable in $\mathrm{TMH}$ iff there is some finite $X^{\prime} \subseteq X$ such that all $\mathrm{TMH}$ trees from $X^{\prime}$ to $C$ are closed 5 We write the following to express that such an argument is provable in $\mathrm{TMH}$.

$$
X \vdash_{\text {тмн }} C
$$

We now proceed to give a Smullyan-style completeness proof for TMH with respect to $\mathrm{MH}$. We first need the following lemma.

Lemma 2.3.1. Fix any open branch $\mathrm{R}$ of an open, finished $\mathrm{TMH}$ tree. Define the induced interpretation of $\mathrm{R}$ to be the $\mathrm{MH}$ valuation $v_{\mathrm{R}}$ that assigns semantic values to each atomic formula $p$ as follows.

$$
v_{\mathrm{R}}(p)= \begin{cases}1 & \text { if } p \oplus \text { is on } \mathrm{R} \\ 0 & \text { if } \neg p \oplus \text { is on } \mathrm{R} \\ \frac{1}{2} & \text { otherwise }\end{cases}
$$

Since $\mathrm{R}$ is open, at least one of each pair of positively signed literals, $p \oplus$ and $\neg p \oplus$, does not occur on the branch. So, the valuation is well-defined. By induction on formula complexity, we show that for all formulas $A$ :

$$
v_{\mathrm{R}}(A) \in \mathbb{D} \text { if } A \oplus \text { is on } \mathrm{R} \text { and } v_{\mathrm{R}}(A) \notin \mathbb{D} \text { if } A \ominus \text { is on } \mathrm{R}
$$

\section{Proof.}

(I) Base case: $A$ is some atomic formula $p$.

\footnotetext{
${ }^{5}$ In such a system, if one tree is closed, any finished tree with the same root is also closed.
}

Australasian Journal of Logic (14:1) 2017, Article no. 11 
(a) Suppose $p \oplus$ is on $\mathrm{R}$. Then $v_{\mathrm{R}}(p) \in \mathbb{D}$ by definition of $v_{\mathrm{R}}$.

(b) Suppose $p \ominus$ is on R. Then, since $\mathrm{R}$ is open, $p \oplus$ is not on R. If $\neg p \oplus$ is on $\mathrm{R}$, then $v_{\mathrm{R}}(p)=0$ by definition of $v_{\mathrm{R}}$. If $\neg p \oplus$ is not on $\mathrm{R}$, then $v_{\mathrm{R}}(p)=\frac{1}{2}$ by definition of $v_{\mathrm{R}}$. Either way, $v_{\mathrm{R}}(p) \notin \mathbb{D}$.

(II) First inductive case: $A$ is a formula of the form $\neg B$.

(a) Suppose $B$ is some atomic $p$.

i. Suppose $\neg p \oplus$ is on R. Then $v_{\mathrm{R}}(p)=0$ by definition of $v_{\mathrm{R}}$, hence $v_{\mathrm{R}}(\neg p) \in \mathbb{D}$.

ii. Suppose $\neg p \ominus$ is on $\mathrm{R}$. Then, since $\mathrm{R}$ is open, $\neg p \oplus$ is not on $\mathrm{R}$. If $p \oplus$ is on $\mathrm{R}$, then $v_{\mathrm{R}}(p)=1$ by definition of $v_{\mathrm{R}}$. If $p \oplus$ is not on $\mathrm{R}$, then $v_{\mathrm{R}}(p)=\frac{1}{2}$ by definition of $v_{\mathrm{R}}$. Either way, $v_{\mathrm{R}}(p) \neq 0$ and so $v_{\mathrm{R}}(\neg p) \notin \mathbb{D}$.

(b) Suppose $B$ is itself a complex formula of the form $\neg C$.

i. Suppose $\neg \neg C \oplus$ is on R. Then, since the tree is finished, $C \oplus$ is on $\mathrm{R}$. So, $v_{\mathrm{R}}(C) \in \mathbb{D}$ by IH, hence $v_{\mathrm{R}}(\neg \neg C) \in \mathbb{D}$.

ii. Suppose $\neg \neg C \ominus$ is on R. Then, since the tree is finished, $C \ominus$ is on $\mathrm{R}$. So, $v_{\mathrm{R}}(C) \notin \mathbb{D}$ by IH, hence $v_{\mathrm{R}}(\neg \neg C) \notin \mathbb{D}$.

(c) Suppose $B$ is a formula of the form $C \wedge D$.

i. Suppose $\neg(C \wedge D) \oplus$ is on $\mathrm{R}$. Then, since the tree is finished, either $\neg C \oplus$ is on $\mathrm{R}$ or $\neg D \oplus$ is on $\mathrm{R}$. So, either $v_{\mathrm{R}}(\neg C) \in \mathbb{D}$ or $v_{\mathrm{R}}(\neg D) \in \mathbb{D}$ by $\mathrm{IH}$, and so either $v_{\mathrm{R}}(C)=0$ or $v_{\mathrm{R}}(D)=0$, hence $v_{\mathrm{R}}(\neg(C \wedge D)) \in \mathbb{D}$.

ii. Suppose $\neg(C \wedge D) \ominus$ is on R. Then, since the tree is finished, both $\neg C \ominus$ and $\neg D \ominus$ are on R. So, both $v_{\mathrm{R}}(\neg C) \notin \mathbb{D}$ and $v_{\mathrm{R}}(\neg D) \notin \mathbb{D}$ by $\mathrm{IH}$, and so both $v_{\mathrm{R}}(C) \neq 0$ and $v_{\mathrm{R}}(D) \neq 0$, hence $v_{\mathrm{R}}(\neg(C \wedge D))=0$, i.e. $v_{\mathrm{R}}(\neg(C \wedge D)) \notin \mathbb{D}$.

(d) Suppose $B$ is a formula of the form $C \vee D$.

i. Suppose $\neg(C \vee D) \oplus$ is on $\mathrm{R}$. Then, since the tree is finished, either (1) all of $C \ominus$ and $\neg C \ominus$ and $D \ominus$ and $\neg D \ominus$ are on $\mathrm{R}$ or else (2) $\neg C \oplus$ and $\neg D \oplus$ are on R. So, by IH we have that either $(1) v_{\mathrm{R}}(C) \notin \mathbb{D}$ and $v_{\mathrm{R}}(\neg C) \notin \mathbb{D}$ and $v_{\mathrm{R}}(D) \notin \mathbb{D}$ and $v_{\mathrm{R}}(\neg D) \notin \mathbb{D}$ in which case $v_{\mathrm{R}}(C)=v_{\mathrm{R}}(D)=\frac{1}{2}$ or else $(2) v_{\mathrm{R}}(\neg C) \in \mathbb{D}$ and $v_{\mathrm{R}}(\neg D) \in \mathbb{D}$ in which case $v_{\mathrm{R}}(C)=v_{\mathrm{R}}(D)=0$. Either way, $v_{\mathrm{R}}(C \vee D)=0$ and so $v_{\mathrm{R}}(\neg(C \vee D)) \in \mathbb{D}$. 
ii. Suppose $\neg(C \vee D) \ominus$ is on $\mathrm{R}$. Then, since the tree is finished, either (1) $C \oplus$ is on R or (2) $D \oplus$ is on R or (3) all of $C \ominus$ and $\neg C \ominus$ and $\neg D \oplus$ are on R or (4) all of $D \ominus$ and $\neg D \ominus$ and $\neg C \oplus$ are on R. So, by IH we have that either $(1) v_{\mathrm{R}}(C) \in \mathbb{D}$ or $(2) v_{\mathrm{R}}(D) \in \mathbb{D}$ or $(3) v_{\mathrm{R}}(C) \notin \mathbb{D}$ and $v_{\mathrm{R}}(\neg C) \notin \mathbb{D}$ and $v_{\mathrm{R}}(\neg D) \in \mathbb{D}$ in which case $v_{\mathrm{R}}(C)=\frac{1}{2}$ and $v_{\mathrm{R}}(D)=0$ or $(4)$ $v_{\mathrm{R}}(D) \notin \mathbb{D}$ and $v_{\mathrm{R}}(\neg D) \notin \mathbb{D}$ and $v_{\mathrm{R}}(\neg C) \in \mathbb{D}$ in which case $v_{\mathrm{R}}(D)=\frac{1}{2}$ and $v_{\mathrm{R}}(C)=0$. Either way, $v_{\mathrm{R}}(C \vee D) \neq 0$ and so $v_{\mathrm{R}}(\neg(C \vee D)) \notin \mathbb{D}$.

(e) Suppose $B$ is a formula of the form $C \rightarrow D$.

i. Suppose $\neg(C \rightarrow D) \oplus$ is on $\mathrm{R}$. Then, since the tree is finished, both $C \oplus$ and $D \ominus$ are on R. So, both $v_{\mathrm{R}}(C) \in \mathbb{D}$ and $v_{\mathrm{R}}(D) \notin$ $\mathbb{D}$ by $\mathrm{IH}$, hence $v_{\mathrm{R}}(C \rightarrow D)=0$ and $v_{\mathrm{R}}(\neg(C \rightarrow D)) \in \mathbb{D}$.

ii. Suppose $\neg(C \rightarrow D) \ominus$ is on R. Then, since the tree is finished, either $C \ominus$ is on $\mathrm{R}$ or $D \oplus$ is on R. So, either $v_{\mathrm{R}}(C) \notin \mathbb{D}$ or $v_{\mathrm{R}}(D) \in \mathbb{D}$ by $\mathrm{IH}$, hence $v_{\mathrm{R}}(C \rightarrow D)=1$ and $v_{\mathrm{R}}(\neg(C \rightarrow$ $D)) \notin \mathbb{D}$.

(III) Second inductive case: $A$ is a formula of the form $B \wedge C$.

(a) Suppose $(B \wedge C) \oplus$ is on $\mathrm{R}$. Then, since the tree is finished, both $B \oplus$ and $C \oplus$ are on R. So, both $v_{\mathrm{R}}(B) \in \mathbb{D}$ and $v_{\mathrm{R}}(C) \in \mathbb{D}$ by $\mathrm{IH}$, hence $v_{\mathrm{R}}(B \wedge C) \in \mathbb{D}$.

(b) Suppose $(B \wedge C) \ominus$ is on $\mathrm{R}$. Then, since the tree is finished, either $B \ominus$ is on $\mathrm{R}$ or $C \ominus$ is on R. So, either $v_{\mathrm{R}}(B) \notin \mathbb{D}$ or $v_{\mathrm{R}}(C) \notin \mathbb{D}$ by $\mathrm{IH}$, hence $v_{\mathrm{R}}(B \wedge C) \notin \mathbb{D}$.

(IV) Third inductive case: $A$ is a formula of the form $B \vee C$.

(a) Suppose $(B \vee C) \oplus$ is on $\mathrm{R}$. Then, since the tree is finished, either $B \oplus$ is on $\mathrm{R}$ or $C \oplus$ is on R. So, either $v_{\mathrm{R}}(B) \in \mathbb{D}$ or $v_{\mathrm{R}}(C) \in \mathbb{D}$ by $\mathrm{IH}$, hence $v_{\mathrm{R}}(B \vee C) \in \mathbb{D}$.

(b) Suppose $(B \vee C) \ominus$ is on $\mathrm{R}$. Then, since the tree is finished, both $B \ominus$ and $C \ominus$ are on R. So, both $v_{\mathrm{R}}(B) \notin \mathbb{D}$ and $v_{\mathrm{R}}(C) \notin \mathbb{D}$ by IH, hence $v_{\mathrm{R}}(B \vee C) \notin \mathbb{D}$.

(V) Fourth inductive case: $A$ is a formula of the form $B \rightarrow C$.

(a) Suppose $(B \rightarrow C) \oplus$ is on $\mathrm{R}$. Then, since the tree is finished, either $B \ominus$ is on $\mathrm{R}$ or $C \oplus$ is on R. So, either $v_{\mathrm{R}}(B) \notin \mathbb{D}$ or $v_{\mathrm{R}}(C) \in \mathbb{D}$ by $\mathrm{IH}$, hence $v_{\mathrm{R}}(B \rightarrow C) \in \mathbb{D}$. 
(b) Suppose $(B \rightarrow C) \ominus$ is on $\mathrm{R}$. Then, since the tree is finished, both $B \oplus$ and $C \ominus$ are on R. So, both $v_{\mathrm{R}}(B) \in \mathbb{D}$ and $v_{\mathrm{R}}(C) \notin \mathbb{D}$ by IH, hence $v_{\mathrm{R}}(B \rightarrow C) \notin \mathbb{D}$.

Theorem 2.3.2 (Completeness of TMH). If $X \vDash_{\mathrm{MH}} A$, then $X \vdash_{\mathrm{TMH}} A$.

Proof. Let $X \nvdash_{\mathrm{TMH}} A$. Fix any finite $X^{\prime} \subseteq X$. Then there is an open TMH tree from $X^{\prime}$ to $A$ by definition of provability in TMH. Fix any open branch $\mathrm{R}$ of this tree with its induced interpretation $v_{\mathrm{R}}$. Since the initial list is the root of every branch, we have $B \oplus$ on $\mathrm{R}$ for all $B \in X^{\prime}$ and we have $A \ominus$ on R. It follows by Lemma 2.3.1 that $v_{\mathrm{R}}(B) \in \mathbb{D}$ for all $B \in X^{\prime}$ and $v_{\mathrm{R}}(A) \notin \mathbb{D}$, hence $X^{\prime} \nvdash_{\mathrm{MH}} A$. Generalizing, there is no finite $X^{\prime} \subseteq X$ such that $X^{\prime} \vDash_{\mathrm{MH}} A$. Thus, $X \nvdash_{\mathrm{MH}} A$ by the compactness of $\mathrm{MH}$.

Theorem 2.3.3 (Soundness of TMH). If $X \vdash_{\mathrm{TMH}} A$, then $X \vDash_{\mathrm{MH}} A$.

Proof Sketch. The soundness of our tableaux system is easier to establish. The proof makes use of a small lemma. For any branch $\mathrm{R}$ of a TMH tree, define a faithful interpretation to be an $\mathrm{MH}$ valuation $v$ that assigns $v(A) \in \mathbb{D}$ if $A \oplus$ is on $\mathrm{R}$ and $v(A) \notin \mathbb{D}$ if $A \ominus$ is on $\mathrm{R}$. We can show that if $v$ is faithful to any initial segment of a branch, when an expansion rule is applied, $v$ is faithful to at least one extension of that segment, and therefore to one branch with that segment. We infer soundness from this as follows. Suppose that $X \nvdash_{\mathrm{MH}} A$. Then there is an $\mathrm{MH}$ valuation $v$ such that $v(B) \in \mathbb{D}$ for all $B \in X$ but $v(A) \notin \mathbb{D}$. Fix any finite $X^{\prime} \subseteq X$ and any finished TMH tree from $X^{\prime}$ to $A$. By definition $v$ is faithful to the root of this tree, so it is faithful to at least one entire branch $\mathrm{R}$ of the tree. If $\mathrm{R}$ were a closed branch, it would either have $A \oplus$ and $A \ominus$ on it for some formula $A$, or it would have $A \oplus$ and $\neg A \oplus$ on it for some formula $A$. But this is impossible as it requires either that $v(A) \in \mathbb{D}$ and $v(A) \notin \mathbb{D}$, or that $v(A) \in \mathbb{D}$ and $v(\neg A) \in \mathbb{D}$. Generalizing, there is no finite $X^{\prime} \subseteq X$ such that all TMH trees from $X^{\prime}$ to $A$ are closed. Therefore, $X \nvdash_{\mathrm{TMH}} A$.

This demonstrates the adequacy of $\mathrm{TMH}$ with respect to $\mathrm{MH}$.

\section{Paraconsistent Hybrid Logic NH}

\subsection{Semantics and Noteworthy Features}

In the second half of the paper, we shift our focus to the propositional logic $\mathrm{NH}$. We use the same naming conventions as before. The logic we define in 
this section is paraconsistent but non-paracomplete with a hybrid of features found in the well-known logics LP (Priest, 1979) and $\mathrm{P}^{2}$ (Marcos, 2005) ${ }^{6}$ so we call it $\mathrm{NH}$. The matrix semantics for $\mathrm{NH}$ has the same negation operation as LP and $\mathrm{P}^{2}$, the disjunction of LP, and the implication of $\mathrm{P}^{2}$.

Conjunction, however, has unfamiliar behavior in this logic, so we want to give some brief motivating remarks about its semantics. We take the LP semantics for negation, disjunction, and conjunction as background and argue for a small modification to its treatment of conjunction. This is premised on the supposition that there are violations of explosion. Suppose, then, that it is rationally permissible to accept some contradiction $(A \wedge \neg A)$. That is to say, logic does not, in itself, compel us to reject such a formula. In that case, it is appropriate to call $A$ a locus of rational inconsistency, or more simply an inconsistent formula. On most views, inconsistent formulas are exceptional, arising from quirks of mind and language such as generalization and self-reference. Many formulas $B$ are consistent in the sense that we ought, instead, to accept $\neg(B \wedge \neg B)$ with respect to such formulas. The properties of consistency and inconsistency, so understood, serve a diagnostic semantic role, i.e. inconsistent formulas are exactly those that ground the failure of explosion. Hence, unlike a formula and its negation-which, by hypothesis, can be jointly accepted-regarding a formula to be both consistent and inconsistent seems to be categorically ruled out. One posit that easily accommodates all of these assumptions is that $\neg(A \wedge \neg A)$ ought to be rejected (in model-theoretic terms, be undesignated) just when $A$ is inconsistent. Given the standard three-valued definition of negation, however, that requires that when $A$ is inconsistent, $(A \wedge \neg A)$ is just true. Moreover, if explosion remains valid, then the LP semantics for negation, disjunction, and conjunction is almost wholly correct with one exception: a conjunction of inconsistent formulas is just true. That is the approach of $\mathrm{NH}$.

In less philosophical terms, we can also see this logic as a solution to the engineering problem of da Costa (1974). Suppose we are interested in a particular articulation of the paraconsistent conviction: we want a propositional logic in which explosion is invalid but where assertion of this fact is non-vacuous, viz. a conclusion of the form $\neg(A \wedge \neg A)$ does not necessarily follow from any arbitrary set of premises whatsoever. It also ought to be straightforward to extend the logic with quantifiers, etc. $\mathrm{NH}$ solves this problem. And while it is certainly not the only possible solution, Beziau and Franceschetto (2015, p.137) point out that the type

\footnotetext{
${ }^{6}$ A natural variation on the logic $\mathrm{P}^{1}$ first studied by Sette $(1973)$.
} 
of semantics we use 'minimizes molecularization (molecular propositions behaving classically)'. So, it has a number of attractive purely formal qualities.

The signature of $\mathrm{NH}$ is below, with a set of formulas FORM defined in the usual way.

$$
\left|p_{i}\right| \neg|\wedge| \vee|\rightarrow|
$$

We also introduce a biconditional in the usual way $(A \leftrightarrow B)={ }_{\text {df. }}(A \rightarrow$ $B) \wedge(B \rightarrow A)$. The set of semantic values of $\mathrm{NH}$ is $\mathbb{S}=\left\{1, \frac{1}{2}, 0\right\}$, the set of designated values is $\mathbb{D}=\left\{1, \frac{1}{2}\right\}$, and an $\mathrm{NH}$ valuation is any function $v: F O R M \rightarrow \mathbb{S}$ closed under the following operations.

\begin{tabular}{|c|c|}
\hline$\neg$ & \\
\hline 1 & 0 \\
$\frac{1}{2}$ & $\frac{1}{2}$ \\
0 & 1 \\
\hline
\end{tabular}

\begin{tabular}{|c|ccc|}
\hline$\wedge$ & 1 & $\frac{1}{2}$ & 0 \\
\hline 1 & 1 & $\frac{1}{2}$ & 0 \\
$\frac{1}{2}$ & $\frac{1}{2}$ & 1 & 0 \\
0 & 0 & 0 & 0 \\
\hline
\end{tabular}

\begin{tabular}{|c|ccc|}
\hline$\vee$ & 1 & $\frac{1}{2}$ & 0 \\
\hline 1 & 1 & 1 & 1 \\
$\frac{1}{2}$ & 1 & $\frac{1}{2}$ & $\frac{1}{2}$ \\
0 & 1 & $\frac{1}{2}$ & 0 \\
\hline
\end{tabular}

\begin{tabular}{|c|ccc|}
\hline$\rightarrow$ & 1 & $\frac{1}{2}$ & 0 \\
\hline 1 & 1 & 1 & 0 \\
$\frac{1}{2}$ & 1 & 1 & 0 \\
0 & 1 & 1 & 1 \\
\hline
\end{tabular}

We define the consequence relation of $\mathrm{NH}$ as follows.

$X \vDash_{\mathrm{NH}} C$ iff on all $\mathrm{NH}$ valuations $v$, either $v(A) \notin \mathbb{D}$ for some $A \in X$ or $v(C) \in \mathbb{D}$

Before turning to proof-theoretic matters, we highlight some features of potential philosophical interest in this logic. $\mathrm{NH}$ has a paraconsistent but non-paracomplete negation as exemplified by the failure of explosion (a) and the presence of excluded middle (b). It also has asymmetrically defined conjunction and disjunction connectives for which the customary inferences (c)-(g) hold, but for which only some of the DeMorgan laws hold (h) while others fail (i). The conditional satisfies conditional proof $(\mathrm{j})$ and classical logical equivalents are intersubstitutible $(\mathrm{k})$.

(a) $A \wedge \neg A \not \nvdash_{\mathrm{NH}} B$

(b) $A \vDash_{\mathrm{NH}} B \vee \neg B$

(c) $A \wedge B \vDash_{\mathrm{NH}} A$

(d) $A \vDash_{\mathrm{NH}} B$ and $A \vDash_{\mathrm{NH}} C \Longrightarrow A \vDash_{\mathrm{NH}} B \wedge C$

(e) $A \vDash_{\mathrm{NH}} A \vee B$ 
(f) $A \vDash_{\mathrm{NH}} C$ and $B \vDash_{\mathrm{NH}} C \Longrightarrow A \vee B \vDash_{\mathrm{NH}} C$

(g) $A \wedge(B \vee C) \vDash_{\mathrm{NH}}(A \wedge B) \vee(A \wedge C)$

(h) $\neg A \wedge \neg B \vDash_{\mathrm{NH}} \neg(A \vee B)$

(i) $\neg A \vee \neg B \not \nvdash_{\mathrm{NH}} \neg(A \wedge B)$

(j) $X, A \vDash_{\mathrm{MH}} B \Longrightarrow X \vDash_{\mathrm{MH}} A \rightarrow B$

(k) $A \leftrightarrow B, \neg A \leftrightarrow \neg B \vDash_{\mathrm{MH}} \Psi(A) \leftrightarrow \Psi(B)$

where $\Psi(B)$ is obtained from $\Psi(A)$ by replacing zero or more instances of $A$ with $B$.

The failure of (i) is symptomatic of the fact that, in virtue of its conjunction operation, $\mathrm{NH}$ has the resources to express when a formula is consistent as follows: $v(\neg(A \wedge \neg A)) \in \mathbb{D}$ iff $v(A) \neq \frac{1}{2}$. In many paraconsistent but non-paracomplete logics, it is vacuous to deny a contradiction because any such claim is a tautology. This results from the DeMorgan law that fails to hold in NH. Denying an explicit contradiction is, thus, non-vacuous in $\mathrm{NH}$ because not all premises guarantee the consistency of arbitrary conclusions. As a result, we can define a consistency operator $\circ A={ }_{\text {df. }} \neg(A \wedge \neg A)$ with the following features in $\mathrm{NH}$.

(j) $(A \wedge \neg A) \wedge \mathrm{O} A \vDash_{\mathrm{NH}} B$

(k) $\neg(A \leftrightarrow \neg A) \vDash_{\mathrm{NH}} \circ A$

(1) $\circ A \wedge \circ B \vDash_{\mathrm{NH}} \mathrm{O}(A \wedge B)$

(m) $\circ(A \vee B) \vDash_{\mathrm{NH}} \circ A \vee \circ B$

(n) $\neg A \vee \neg B \vDash_{\mathrm{NH}} \neg(A \wedge B) \vee \neg \mathrm{O}(A \wedge B)$

(o) $A, \neg B, \circ B \vDash_{\mathrm{NH}} \neg(A \rightarrow B)$

(p) $\circ A,(A \rightarrow B) \not \nvdash_{\mathrm{NH}} \neg B \rightarrow \neg A$

If we think of $O$ as expressing rational consistency, i.e. that one must not both assert and deny such formulas despite the possibility of nontrivially holding other inconsistent commitments, then most of these laws are intuitively correct. We have a law of 'consistent explosion' that says that no formula can, inconsistently, be both asserted and denied when it is also held to be consistent $(\mathrm{j})$. We get a near-cousin of our missing DeMorgan law (n), which arises from the formal strategy of $\mathrm{NH}$, viz. to extract expressive 
power from a novel treatment of conjunction. The formulas with respect to which a theory is consistent are those that are inequivalent to their own negations (k) and consistency restricts contraposition (o)-(p).

Our conditional generalizes material implication, insofar as a conditional formula is true just when its antecedent is undesignated or its consequent is designated. As a result, $\mathrm{NH}$ validates stronger implicational forms of reasoning than LP, such as modus ponens, as well as genuinely trivializing entailments, such as $\neg(A \rightarrow A) \vDash_{\mathrm{NH}} B$. It has the rule of conditional weakening $A \vDash_{\mathrm{MH}} B \rightarrow A$ which fails even in the three-valued almostrelevant logic $\mathrm{RM}_{3}$. So it is a strong paraconsistent logic. On the other hand, certain other inferences fail in $\mathrm{NH}$, such as $\neg A \wedge \neg B \nvdash_{\mathrm{NH}} \neg((A \rightarrow B) \rightarrow B)$, but which hold in $\mathrm{RM}_{3}$ Although $\mathrm{NH}$ and $\mathrm{RM}_{3}$ each strictly extends Priest's logic of paradox, neither is properly contained in the other. $\mathrm{NH}$ also, notably, respects the intersubstitutivity of $\bigcirc A$ and $\bigcirc \neg A$ for any pair of contradictories $A$ and $\neg A$, which notoriously fails in DaCosta's hierarchy of paraconsistent logics $]^{7}$ Having thus situated $\mathrm{NH}$ in the logical terrain, we turn to its proof theory.

\subsection{Hilbert System HNH}

In this subsection, we develop a Hilbert system $\mathrm{HNH}$ and show that it is sound and complete for our paraconsistent hybrid logic NH. Any uniform substitution of formulas into metavariable positions in one of the following schemas is an axiom of HNH. We note that (Ax.1)-(Ax.9) are common to both of our Hilbert systems.

(Ax.1) $A \rightarrow(B \rightarrow A)$

(Ax.2) $(A \rightarrow(B \rightarrow C)) \rightarrow((A \rightarrow B) \rightarrow(A \rightarrow C))$

(Ax.3) $(A \wedge B) \rightarrow A$

(Ax.4) $(A \wedge B) \rightarrow B$

$(\mathbf{A x . 5 )}(A \rightarrow B) \rightarrow((A \rightarrow C) \rightarrow(A \rightarrow(B \wedge C)))$

(Ax.6) $A \rightarrow(A \vee B)$

(Ax.7) $B \rightarrow(A \vee B)$

$(\mathbf{A x . 8 )}(A \rightarrow C) \rightarrow((B \rightarrow C) \rightarrow((A \vee B) \rightarrow C))$

\footnotetext{
${ }^{7}$ See Marcos (2005, p.56).
} 
(Ax.9) $\neg \neg A \leftrightarrow A$

(Ax.17) $\bigcirc(A \wedge \neg A)$

(Ax.18) $\bigcirc(A \rightarrow B)$

(Ax.19) $\bigcirc A \rightarrow((B \rightarrow A) \rightarrow(\neg A \rightarrow \neg B))$

(Ax.20) $(\neg A \wedge \neg B) \leftrightarrow \neg(A \vee B)$

(Ax.21) $(\neg A \vee \neg B) \leftrightarrow(\neg(A \wedge B) \vee((A \wedge \neg A) \wedge(B \wedge \neg B)))$

(Ax.22) $(\circ A \wedge \neg A) \rightarrow(A \rightarrow B)$

(Ax.23) $(A \wedge(\mathrm{O} B \wedge \neg B)) \rightarrow \neg(A \rightarrow B)$

We also have a rule of modus ponens.

$$
(\mathbf{M P}) A \rightarrow B, A \Longrightarrow B
$$

A derivation in $\mathrm{HNH}$ from the set of assumptions $X$ to conclusion $C$ is a finite sequence of formulas $A_{1}, \ldots, A_{n}$ such that $A_{n}=C$ and such that every $A_{i}$ in the sequence is either a member of $X$, an axiom of $\mathrm{HNH}$, or follows from previous formulas in the sequence by MP. We write the following to express that such a derivation exists.

$$
X \vdash_{\mathrm{HNH}} C
$$

A formula that is derivable from the empty set is a theorem of $\mathrm{HNH}$.

$\mathrm{HNH}$ has a deduction theorem because it contains the full axioms of intuitionistic implication. It also has a law of excluded middle and a law reflecting the fact that 'consistency' is fixed over negation. We note several other useful theorems and derived rules below. These results are established in the appendix.

(Iden) $A \rightarrow A$

(CFN) $\bigcirc A \leftrightarrow \circ \neg A$

(NEX) $\bigcirc A \rightarrow((A \wedge \neg A) \rightarrow B)$

(Dial) $\neg \mathrm{O} A \rightarrow A$ and $\neg \mathrm{O} A \rightarrow \neg A$

(Adj) $A, B \Longrightarrow A \wedge B$ 
(Tran) $A \rightarrow B, B \rightarrow C \Longrightarrow A \rightarrow C$

(Perm) $A \rightarrow(B \rightarrow C) \Longrightarrow B \rightarrow(A \rightarrow C)$

(NRed) $A \rightarrow \neg A \Longrightarrow \neg A$

(ConC) $\circ(A \wedge B) \Longrightarrow(A \wedge B) \rightarrow(\circ A \vee \neg \mathrm{O} B)$ and $\circ(A \wedge B) \Longrightarrow \neg(A \wedge B) \rightarrow((\neg A \wedge \circ A) \vee(\neg B \wedge \circ B))$

$($ ConD $) \circ(A \vee B) \Longrightarrow(A \vee B) \rightarrow((A \wedge \circ A) \vee(B \wedge \circ B))$ and $\circ(A \vee B) \Longrightarrow \neg(A \vee B) \rightarrow((\neg A \wedge \circ A) \wedge(\neg B \wedge \circ B))$

As with our previous axiom system, and again following the example of Goldberg et al. (1974), we give a Henkin-style completeness proof for $\mathrm{HNH}$ with respect to $\mathrm{NH}$. We need a few definitions and lemmas.

- A set of formulas $X$ is $\mathrm{HNH}$-trivial iff $X \vdash_{\mathrm{HNH}} A$ for all $A$; it is $\mathrm{HNH}$ non-trivial otherwise.

- A set of formulas $X$ is maximal $\mathrm{HNH}$-non-trivial iff it is $\mathrm{HNH}$-nontrivial and $X \cup\{B\}$ is $\mathrm{HNH}$-trivial for all formulas $B \notin X$.

- An $\mathrm{HNH}$-filter is a set of formulas that contains all $\mathrm{NH}$ axioms and is closed under MP.

Lemma 3.2.1. If $X$ is maximal $\mathrm{HNH}$-non-trivial, then $X$ is an $\mathrm{HNH}$-filter.

The can be proven just as it was in Lemma 2.2.1. A maximal HNH-nontrivial set contains all theorems and is closed under derivability, e.g. such sets are closed under adjunction and transitivity. We show that they are also prime.

Corollary (Primeness). If $X$ is maximal $\mathrm{HNH}$-non-trivial, then $X$ is prime; in particular, with respect to any formula $C$, exactly one of $C, \circ C \in X$ or $\neg C, \mathrm{o} C \in X$ or $C, \neg C \in X$.

Proof. The first part runs as before: if $X$ is maximal $\mathrm{HNH}$-non-trivial with $(A \vee B) \in X$, then having both $A \notin X$ and $B \notin X$ is impossible on pain of $X$ 's triviality. In particular, if $X$ is maximal $\mathrm{HNH}$-non-trivial, then with respect to any formula $C$, either $C$ or $\neg C \in X$ and either $\circ C \in X$ or $\neg \mathrm{O} C \in X$ by Lemma 3.2.1 and LEM. This gives us four possibilities: both $C, \circ C \in X$ or both $\neg C, \circ C \in X$ or both $C, \neg \circ C \in X$ or both $\neg C, \neg \mathrm{O} C \in X$. But the last two cases are equivalent to the inconsistent 
case $C, \neg C \in X$ by the definition of $\circ$ and Ax.9. We can have at most one of $C, \circ C \in X$ or $\neg C, \circ C \in X$ or $C, \neg C \in X$ because any combination would be trivializing by adjunction, Ax.22, and NEX.

The next two lemmas will help simplify the rest of the presentation.

Lemma 3.2.2. If $X$ is maximal $\mathrm{HNH}$-non-trivial and $\mathrm{o}(A \wedge B) \in X$, then if $(A \wedge B) \in X$ we either have all of $A, \circ A, B, \circ B \in X$ or all of $A, \neg A, B, \neg B \in X$, and if $\neg(A \wedge B) \in X$ we have both $\neg A, \circ A \in X$ or both $\neg B, \circ B \in X$.

Proof. Fix maximal HNH-non-trivial set $X$ with $\mathrm{O}(A \wedge B) \in X$. Suppose that $(A \wedge B) \in X$. Then both $A, B \in X$ by simplification and

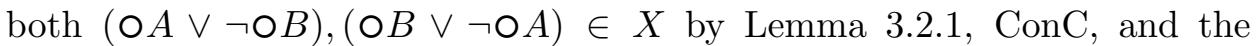
commutativity of conjunction. Then by primeness and non-triviality, either we have both $\circ A, \circ B \in X$ or we have both $\neg \mathrm{O} A, \neg \mathrm{O} B \in X$ which suffices for $\neg A, \neg B \in X$. So, we either have all of $A, \circ A, B, \circ B \in X$ or all of $A, \neg A, B, \neg B \in X$. On the other hand, suppose that $\neg(A \wedge B) \in X$. Then $(\neg A \wedge \circ A) \vee(\neg B \wedge \mathrm{O} B) \in X$ by Lemma 3.2.1 and ConC, so we have both $\neg A, \circ A \in X$ or both $\neg B, \circ B \in X$ by primeness and simplification.

Lemma 3.2.3. If $X$ is maximal $\mathrm{HNH}$-non-trivial and $\mathrm{O}(A \vee B) \in X$, then if $(A \vee B) \in X$ we either have both $A, \circ A \in X$ or both $B, \circ B \in X$, and if $\neg(A \vee B) \in X$ we have all of $\neg A, \circ A, \neg B, \circ B \in X$.

Proof. Fix maximal HNH-non-trivial set $X$ with $\mathrm{O}(A \vee B) \in X$. Suppose that $(A \vee B) \in X$. Then $((A \wedge \circ A) \vee(B \wedge \circ B)) \in X$ by Lemma 3.2.1 and ConD, so either we have both $A, \circ A \in X$ or both $B, \circ B \in X$ by primeness and simplification. On the other hand, suppose that $\neg(A \vee B) \in X$. Then we have all of $\neg A, \circ A, \neg B, \circ B \in X$ by Lemma 3.2.1. ConD, and simplification.

We can now establish the following important relationship.

Lemma 3.2.4. If $X$ is $\mathrm{HNH}$-non-trivial, then $X$ is satisfied on some $\mathrm{NH}$ valuation.

Proof. Fix any HNH-non-trivial set of formulas $X$. We want to extend this set to a maximal $\mathrm{HNH}$-non-trivial superset $X^{*}$. Let $A_{1}, \ldots, A_{n}$ be an enumeration of FORM and define $X^{*}$ recursively as follows.

$$
X_{0}=X
$$




$$
\begin{gathered}
X_{n+1}= \begin{cases}X_{n} \cup\left\{A_{n}\right\} & \text { if this is HNH-non-trivial } \\
X_{n} & \text { otherwise }\end{cases} \\
X^{*}=\bigcup_{n \in \mathbb{N}} X_{n}
\end{gathered}
$$

Succession preserves non-triviality by design, so each of the $X_{i}$ for $i \in \mathbb{N}$ is $\mathrm{HMH}$-non-trivial. In that case, the limit $X^{*}$ must be $\mathrm{HMH}$-non-trivial as well. Otherwise we would have $X^{*} \vdash_{\mathrm{HMH}} \mathrm{O} p \wedge(p \wedge \neg p)$ and, since derivations are finite, that would require $X_{i} \vdash_{\mathrm{HMH}} \mathrm{O} p \wedge(p \wedge \neg p)$ for some $X_{i}$, in which case that $X_{i}$ would be trivial by NEX.

Fix an $\mathrm{NH}$ valuation $v_{X^{*}}$ defined as follows for each atomic formula $p$. By the established primeness of any maximal $\mathrm{HNH}$-non-trivial set, this is a well-defined valuation.

$$
v_{X^{*}}(p)=\left\{\begin{array}{lll}
1 & \text { if } & \text { both } p, \mathrm{o} p \in X^{*} \\
0 & \text { if } & \text { both } \neg p, \mathrm{O} p \in X^{*} \\
\frac{1}{2} & \text { if } & \operatorname{both} p, \neg p \in X^{*}
\end{array}\right.
$$

We show that this valuation extends to all formulas $A$ as follows.

$$
v_{X^{*}}(A)=\left\{\begin{array}{lll}
1 & \text { if } & \text { both } A, \circ A \in X^{*} \\
0 & \text { if } & \text { both } \neg A, \circ A \in X^{*} \\
\frac{1}{2} & \text { if } & \operatorname{both} A, \neg A \in X^{*}
\end{array}\right.
$$

(I) Base case: holds by definition.

(II) First inductive case: $A$ is a formula of the form $B \wedge C$.

(a) Suppose that both $A, \circ A \in X^{*}$, i.e. that both $(B \wedge C), \mathrm{O}(B \wedge C) \in$ $X^{*}$. Then we either have all of $B, \circ B, C, \circ C \in X^{*}$ or all of $B, \neg B, C, \neg C \in X^{*}$ by Lemma 3.2.2. If $B, \circ B, C, \circ C \in X^{*}$, then $v_{X^{*}}(B)=v_{X^{*}}(C)=1$ by IH. If $B, \neg B, C, \neg C \in X^{*}$, then $v_{X^{*}}(B)=v_{X^{*}}(C)=\frac{1}{2}$ by IH. Thus, in every case, $v_{X^{*}}(B \vee C)=$ $v_{X^{*}}(A)=1$.

(b) Suppose that both $\neg A, \circ A \in X^{*}$, i.e. that both $\neg(B \wedge C), \circ(B \wedge$ $C) \in X^{*}$. Then either we have both $\neg B, \circ B \in X^{*}$ or both $\neg C, \circ C \in X^{*}$ by Lemma 3.2.2. If $\neg B, \circ B \in X^{*}$, then $v_{X^{*}}(B)=$ 0 by IH. If $\neg C, \circ C \in X^{*}$, then $v_{X^{*}}(C)=0$ by IH. Thus, in every case, $v_{X^{*}}(B \vee C)=v_{X^{*}}(A)=0$. 
(c) Suppose that both $A, \neg A \in X^{*}$, i.e. that both $(B \wedge C), \neg(B \wedge C) \in$ $X^{*}$. Then by simplification both $B, C \in X^{*}$ and by Ax.21 we have $(\neg B \vee \neg C) \in X^{*}$. We know that either $\circ B \in X^{*}$ or $\neg \mathrm{O} B \in X^{*}$ and either $\circ C \in X^{*}$ or $\neg \mathrm{O} C \in X^{*}$ by LEM. If $\circ B \in X^{*}$, then $(\circ B \wedge \neg \neg B) \in X^{*}$ by Ax.9 and adjunction, and so $(\neg B \rightarrow \neg C) \in X^{*}$ by Ax.22, hence $((\neg B \vee \neg C) \rightarrow \neg C) \in X^{*}$ by Iden and Ax.8, therefore $\neg C \in X^{*}$. In that case, $v_{X^{*}}(B)=1$ and $v_{X^{*}}(C)=\frac{1}{2}$ by IH. If $\circ C \in X^{*}$, then by similar reasoning to above $\neg B \in X^{*}$ and so $v_{X^{*}}(B)=\frac{1}{2}$ and $v_{X^{*}}(C)=1$ by IH. If both $\neg \mathrm{O} B, \neg \mathrm{O} C \in X^{*}$, then both $\neg B, \neg C \in X^{*}$ by Dial and so $v_{X^{*}}(B)=v_{X^{*}}(C)=\frac{1}{2}$ by IH. Thus, in every case, $v_{X^{*}}(B \wedge C)=v_{X^{*}}(A)=\frac{1}{2}$.

(III) Second inductive case: $A$ is a formula of the form $B \vee C$.

(a) Suppose that both $A, \circ A \in X^{*}$, i.e. that both $(B \vee C), \circ(B \vee C) \in$ $X^{*}$. Then either we have both $A, \circ A \in X^{*}$ or both $B, \circ B \in X^{*}$ by Lemma 3.2.3. If both $A, \circ A \in X^{*}$, then $v_{X^{*}}(A)=1$ by IH, hence $v_{X^{*}}(B \vee C)=v_{X^{*}}(A)=1$. Likewise if both $B, \circ B \in X^{*}$.

(b) Suppose that both $\neg A, \mathrm{O} A \in X^{*}$, i.e. that both $\neg(B \vee C), \mathrm{O}(B \vee$ $C) \in X^{*}$. Then we have all of $\neg A, \circ A, \neg B, \circ B \in X^{*}$ by Lemma 3.2 .3 and so $\neg A, \circ A, \neg B, \circ B \in X^{*}$. Hence, $v_{X^{*}}(A)=v_{X^{*}}(B)=$ 0 by $\mathrm{IH}$, hence $v_{X^{*}}(B \vee C)=v_{X^{*}}(A)=0$.

(c) Suppose that both $A, \neg A \in X^{*}$, i.e. that both $(B \vee C), \neg(B \vee C) \in$ $X^{*}$. Then by Ax.20 and simplification, both $\neg B \in X^{*}$ and $\neg C \in X^{*}$. We know that either $\circ B \in X^{*}$ or $\neg \circ B \in X^{*}$ and either $\circ C \in X^{*}$ or $\neg \circ C \in X^{*}$ by LEM. If $\circ B \in X^{*}$, then $(B \rightarrow C) \in X^{*}$ by Ax.22 and so $((B \vee C) \rightarrow C) \in X^{*}$ by Iden and Ax.8, thus $C \in X^{*}$. In that case, $v_{X^{*}}(B)=0$ and $v_{X^{*}}(C)=\frac{1}{2}$ by IH. If $\circ C \in X^{*}$, then by similar reasoning to above $B \in X^{*}$ and so $v_{X^{*}}(B)=\frac{1}{2}$ and $v_{X^{*}}(C)=0$ by IH. If both $\neg \mathrm{O} B, \neg \mathrm{O} C \in X^{*}$, then both $B, C \in X^{*}$ by Dial and so $v_{X^{*}}(B)=v_{X^{*}}(C)=\frac{1}{2}$ by IH. Thus, in every case, $v_{X^{*}}(B \vee C)=v_{X^{*}}(A)=\frac{1}{2}$.

(IV) Third inductive case: $A$ is a formula of the form $B \rightarrow C$.

(a) Suppose that both $A, \mathrm{O} A \in X^{*}$, i.e. that both $(B \rightarrow C), \mathrm{O}(B \rightarrow$ $C) \in X^{*}$. Then $\neg(B \rightarrow C) \rightarrow \neg((\neg \circ B \vee B) \wedge(\circ C \wedge \neg C)) \in X^{*}$ by Ax.1 and Ax.19. We have $(\neg \circ B \vee B) \rightarrow B \in X^{*}$ by Dial, Iden, and Ax.8, so $((\neg \circ B \vee B) \wedge(\circ C \wedge \neg C)) \rightarrow(B \rightarrow C) \in X^{*}$ by 
Ax.23. Hence, $((\neg \mathrm{O} B \vee B) \wedge(\mathrm{O} C \wedge \neg C)) \rightarrow \neg((\neg \mathrm{O} B \vee B) \wedge(\mathrm{O} C \wedge$ $\neg C)) \in X^{*}$ by transitivity. Then $\neg((\neg \mathrm{O} B \vee B) \wedge(\circ C \wedge \neg C)) \in X^{*}$ by NRed, which guarantees that either we have $\neg(\neg \mathrm{O} B \vee B) \in X^{*}$ or $\neg(\circ C \wedge \neg C) \in X^{*}$. In the first case, by Ax.20 and Ax.9 we have both $\circ B, \neg B \in X^{*}$ and so $v_{X^{*}}(B) \notin D$. In the second case, by Ax.6, Ax.21, Dial, Iden, and Ax.8, we have $C \in X^{*}$, and so, regardless of whether $\circ C \in X^{*}$ or $\neg \circ C \in X^{*}$, we have $v_{X^{*}}(C) \in D$. Thus, in every case, $v_{X^{*}}(B \rightarrow C)=v_{X^{*}}(A)=1$.

(b) Suppose that both $\neg A, \circ A \in X^{*}$, i.e. that both $\neg(B \rightarrow$ $C), \mathrm{O}(B \rightarrow C) \in X^{*}$. Then we have $\mathrm{\neg}(B \rightarrow C) \in X^{*}$ by $\mathrm{CFN}$ and so $(B \rightarrow C) \rightarrow \neg((\circ B \wedge \neg B) \vee C) \in X^{*}$ by Ax.1, Ax.19, and Ax.9. We have $((\circ B \wedge \neg B) \vee C) \rightarrow(B \rightarrow C) \in X^{*}$ by Ax.1 and Ax.22 and so $((\circ B \wedge \neg B) \vee C) \rightarrow \neg((\circ B \wedge \neg B) \vee C) \in X^{*}$ by transitivity. Then $\neg((\circ B \wedge \neg B) \vee C) \in X^{*}$ by NRed, which guarantees both $B, \neg C \in X^{*}$ by Ax.20, Ax.21, primeness, and Dial. We know that either $\circ C \in X^{*}$ or $\neg \circ C \in X^{*}$, but the latter trivializes $X^{*}$ by Dial and Ax.1; thus, in fact, we must have $\circ C \in X^{*}$. Then, regardless of whether $\circ B \in X^{*}$ or $\neg \mathrm{O} B \in X^{*}$, we have $v_{X^{*}}(B) \in D$ and $v_{X^{*}}(C) \notin D$ by IH, therefore $v_{X^{*}}(B \rightarrow C)=v_{X^{*}}(A)=0$.

(c) The third option, that both $A, \neg A \in X^{*}$, i.e. both $(B \rightarrow$ $C), \neg(B \rightarrow C) \in X^{*}$, is not really possible. For if it holds, then since $(((B \rightarrow C) \wedge \neg(B \rightarrow C)) \rightarrow D) \in X^{*}$ for all formulas $D$ by Ax.18 and NEX, we would have $D \in X^{*}$ for all $D$ by adjunction and closure, contradicting the non-triviality of $X^{*}$.

(V) Fourth inductive case: $A$ is a formula of the form $\neg B$. In case $B$ is atomic or it has conjunction, disjunction, or implication as its main connective, this is covered by steps (II)b. (III)b, and (IV)b. The remaining case is where $B$ itself is of the form $\neg C$.

(a) Suppose that both $A, \circ A \in X^{*}$, i.e. that both $\neg \neg C, \circ \neg \neg C \in X^{*}$. Then by Ax.9 and CFN both $C, \mathrm{O} C \in X^{*}$ and so, by IH, $v_{X^{*}}(C)=v_{X^{*}}(\neg \neg C)=v_{X^{*}}(A)=1$.

(b) Suppose that both $\neg A, \mathrm{\circ} A \in X^{*}$, i.e. that both $\neg \neg \neg C, \mathrm{O} \neg \neg C \in$ $X^{*}$. Then by Ax.9 and CFN both $\neg C, \circ C \in X^{*}$ and so, by $\mathrm{IH}$, $v_{X^{*}}(C)=v_{X^{*}}(\neg \neg C)=v_{X^{*}}(A)=0$.

(c) Suppose that both $A, \neg A \in X^{*}$, i.e. that both $\neg \neg C, \neg \neg \neg C \in X^{*}$. Then by Ax.9 and closure both $C, \neg C \in X^{*}$ and so, by IH, $v_{X^{*}}(C)=v_{X^{*}}(\neg \neg C)=v_{X^{*}}(A)=\frac{1}{2}$. 
It follows that $A \in X^{*}$ iff $v_{X^{*}}(A) \in \mathbb{D}$ for all formulas $A$. This shows that $X^{*}$ and any of its subsets, including our original set $X$, are satisfied on some $\mathrm{NH}$ valuation.

This is all that we need to establish completeness for our Hilbert system.

Theorem 3.2.5 (Completeness of $\mathrm{HNH}$ ). If $X \vDash_{\mathrm{NH}} A$, then $X \vdash_{\mathrm{HNH}} A$.

Proof. Suppose that $X \vDash_{\mathrm{NH}} A$. Then there is no NH valuation on which $v(B) \in \mathbb{D}$ for all $B \in X$ and $v(A) \notin \mathbb{D}$. In that case, $X \cup\{\neg A \vee \circ A\}$ is unsatisfied on all $\mathrm{NH}$ valuations. It follows by Lemma 3.2 .4 that $X \cup\{\neg A \vee \mathrm{O} A\}$ is $\mathrm{HNH}$-trivial. Then $X, \neg A \vee \mathrm{O} A \vdash_{\mathrm{HNH}} A$ and so we have $X \vdash_{\text {HNH }} \neg A \rightarrow A$ by RBC and the deduction theorem. Therefore, by NRed and Ax.9 we have $X \vdash_{\text {HNH }} A$.

Theorem 3.2.6 (Soundness of $\mathrm{HNH}$ ). If $X \vdash_{\mathrm{HNH}} A$, then $X \vDash_{\mathrm{NH}} A$.

Proof Sketch. This result is more straightforward. Let $X \vdash_{\mathrm{HNH}} A$ and fix any $\mathrm{HNH}$ derivation from $X$ to $A$ that witnesses the fact. By induction on the length of derivations, we can show that if an $\mathrm{NH}$ valuation $v$ assigns $v(B)=1$ for all $B \in X$, then $v(A)=1$. For the base case, $A$ can only be an $\mathrm{HNH}$ axiom. It is straightforward if tedious to show that every axiom is an $\mathrm{NH}$ tautology. For the inductive case, we only need the fact that if $v(C)=1$ and $v(C \rightarrow D)=1$ then $v(D)=1$, i.e. that MP preserves designation.

This demonstrates the adequacy of $\mathrm{HNH}$ with respect to $\mathrm{NH}$. Since derivations in $\mathrm{HNH}$ are finite, we again get a quick compactness result for $\mathrm{NH}$.

Corollary (Compactness of $\mathrm{NH}) . X \vDash_{\mathrm{NH}} A$ iff $X^{\prime} \vDash_{\mathrm{NH}} A$ for some finite $X^{\prime} \subseteq X$.

\subsection{Tableaux System TNH}

In this subsection, we develop a tableaux system TNH and show that it, too, is sound and complete for $\mathrm{NH}$. As before, our tableaux are downwardbranching trees of signed formulas, using signs $\oplus$ and $\ominus$ for designated and undesignated value.

Given a finite premise set $X$ and conclusion $C$, the root of the TNH tree from $X$ to $C$ is a list consisting of $B \oplus$ for all $B \in X$ and $C \ominus$. Starting from this initial list, we apply the expansion rules below to construct the tree. Apart from the expansion rules, which differ, the construction process for these trees is the same as described in 2.3 


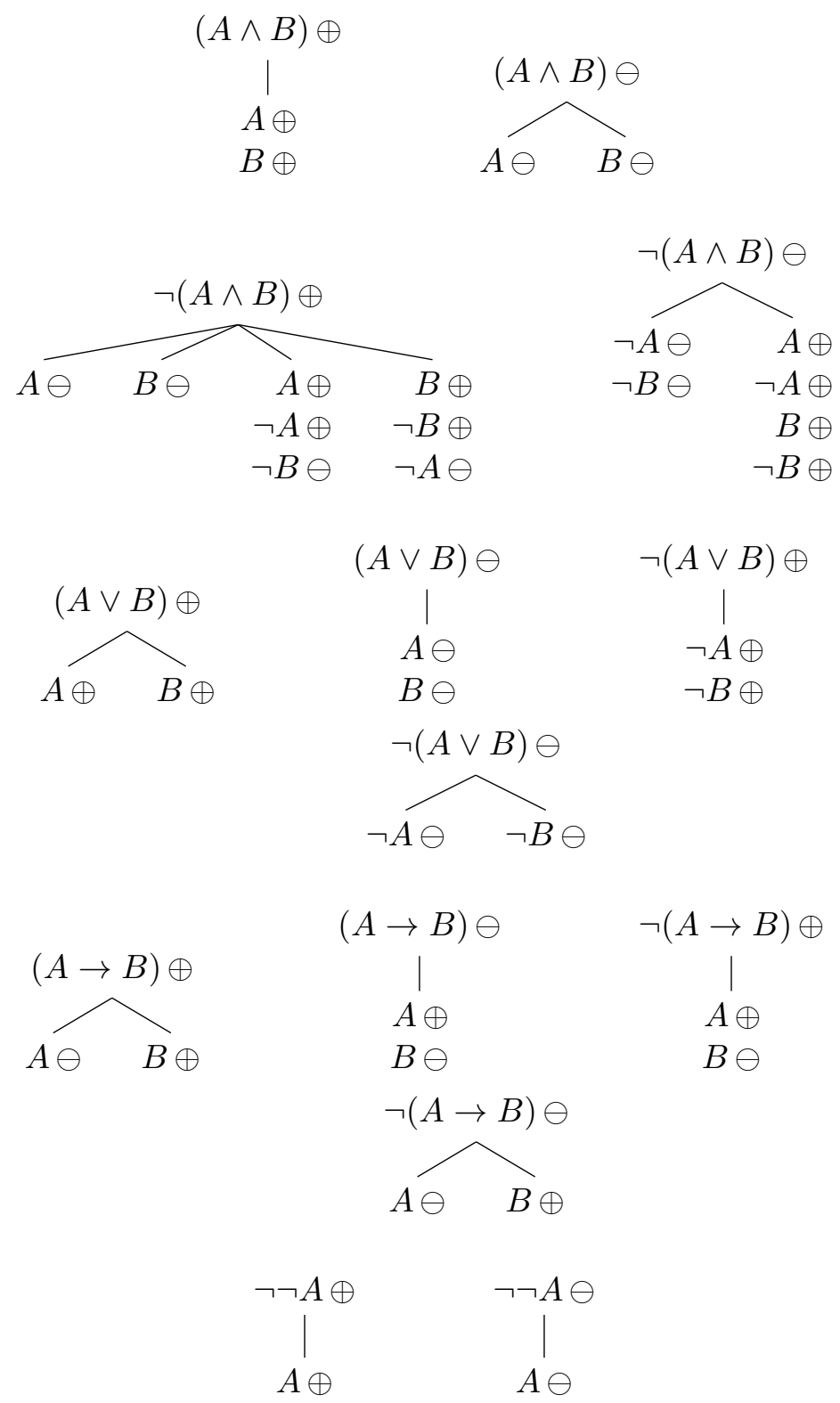

We can think of an expanding branch as a search for an NH countermodel to the original argument. At any stage of the construction, a branch is closed if, for any formula $A$, both $A \oplus$ and $A \ominus$ occur on that branch or both $A \ominus$ and $\neg A \ominus$ occur on that branch. Such a branch has failed to produce a viable counter-model. When every rule that can be applied has

Australasian Journal of Logic (14:1) 2017, Article no. 11 
been applied, the tree is finished. Every argument has a finite, finished TNH tree.

If every branch of a finished tree is closed, the tree is closed; otherwise it is open. The argument from arbitrary premise set $X$ to conclusion $C$ is provable in TNH iff there is some finite $X^{\prime} \subseteq X$ such that all TNH trees from $X^{\prime}$ to $C$ are closed ${ }^{8}$ We write the following to express that such an argument is provable in $\mathrm{TNH}$.

$$
X \vdash_{\mathrm{TNH}} C
$$

We again proceed to give a Smullyan-style completeness proof for TNH with respect to $\mathrm{NH}$. We first need the following lemma.

Lemma 3.3.1. Fix any open branch $\mathrm{R}$ of an open, finished TNH tree. Define the induced interpretation of $\mathrm{R}$ to be the $\mathrm{NH}$ valuation $v_{\mathrm{R}}$ that assigns semantic values to each atomic formula $p$ as follows.

$$
v_{\mathrm{R}}(p)= \begin{cases}1 & \text { if } \neg p \ominus \text { is on } \mathrm{R} \\ 0 & \text { if } p \ominus \text { is on } \mathrm{R} \\ \frac{1}{2} & \text { otherwise }\end{cases}
$$

Since $\mathrm{R}$ is open, at least one of each pair of negatively signed literals, $p \ominus$ and $\neg p \ominus$, does not occur on the branch. So, the valuation is well-defined. By induction on formula complexity, we show that for all formulas $A$ :

$$
v_{\mathrm{R}}(A) \in \mathbb{D} \text { if } A \oplus \text { is on } \mathrm{R} \text { and } v_{\mathrm{R}}(A) \notin \mathbb{D} \text { if } A \ominus \text { is on } \mathrm{R}
$$

\section{Proof.}

(I) Base case: $A$ is some atomic formula $p$.

(a) Suppose $p \oplus$ is on $\mathrm{R}$. Then, since $\mathrm{R}$ is open, $p \ominus$ is not on $\mathrm{R}$. If $\neg p \ominus$ is on $\mathrm{R}$, then $v_{\mathrm{R}}(p)=1$ by definition of $v_{\mathrm{R}}$. If $\neg p \ominus$ is not on $\mathrm{R}$, then $v_{\mathrm{R}}(p)=\frac{1}{2}$ by definition of $v_{\mathrm{R}}$. Either way, $v_{\mathrm{R}}(p) \in \mathbb{D}$.

(b) Suppose $p \ominus$ is on $\mathrm{R}$. Then $v_{\mathrm{R}}(p) \notin \mathbb{D}$ by definition of $v_{\mathrm{R}}$.

(II) First inductive case: $A$ is a formula of the form $\neg B$.

(a) Suppose $B$ is some atomic $p$.

\footnotetext{
${ }^{8}$ In such a system, if one tree is closed, any finished tree with the same root is also closed.
} 
i. Suppose $\neg p \oplus$ is on $\mathrm{R}$. Then, since $\mathrm{R}$ is open, $\neg p \ominus$ is not on $\mathrm{R}$. If $p \ominus$ is on R, then $v_{\mathrm{R}}(p)=0$ by definition of $v_{\mathrm{R}}$. If $p \ominus$ is not on $\mathrm{R}$, then $v_{\mathrm{R}}(p)=\frac{1}{2}$ by definition of $v_{\mathrm{R}}$. Either way, $v_{\mathrm{R}}(p) \neq 1$ and so $v_{\mathrm{R}}(\neg p) \in \mathbb{D}$.

ii. Suppose $\neg p \ominus$ is on R. Then $v_{\mathrm{R}}(p)=1$ by definition of $v_{\mathrm{R}}$, hence $v_{\mathrm{R}}(\neg p) \notin \mathbb{D}$.

(b) Suppose $B$ is itself a complex formula of the form $\neg C$.

i. Suppose $\neg \neg C \oplus$ is on R. Then, since the tree is finished, $C \oplus$ is on $\mathrm{R}$. So, $v_{\mathrm{R}}(C) \in \mathbb{D}$ by IH, hence $v_{\mathrm{R}}(\neg \neg C) \in \mathbb{D}$.

ii. Suppose $\neg \neg C \ominus$ is on R. Then, since the tree is finished, $C \ominus$ is on $\mathrm{R}$. So, $v_{\mathrm{R}}(C) \notin \mathbb{D}$ by $\mathrm{IH}$, hence $v_{\mathrm{R}}(\neg \neg C) \notin \mathbb{D}$.

(c) Suppose $B$ is a formula of the form $C \wedge D$.

i. Suppose $\neg(C \wedge D) \oplus$ is on $\mathrm{R}$. Then, since the tree is finished, either (1) $C \ominus$ is on R or (2) $D \ominus$ is on R or (3) all of $C \oplus$ and $\neg C \oplus$ and $\neg D \ominus$ are on $\mathrm{R}$ or (4) all of $D \oplus$ and $\neg D \oplus$ and $\neg C \ominus$ are on R. So, by IH, we have that either $(1) v_{\mathrm{R}}(C) \notin \mathbb{D}$ or $(2) v_{\mathrm{R}}(D) \notin \mathbb{D}$ or $(3) v_{\mathrm{R}}(C) \in \mathbb{D}$ and $v_{\mathrm{R}}(\neg C) \in \mathbb{D}$ and $v_{\mathrm{R}}(\neg D) \notin \mathbb{D}$ in which case $v_{\mathrm{R}}(C)=\frac{1}{2}$ and $v_{\mathrm{R}}(D)=1$ or $(3)$ $v_{\mathrm{R}}(D) \in \mathbb{D}$ and $v_{\mathrm{R}}(\neg D) \in \mathbb{D}$ and $v_{\mathrm{R}}(\neg C) \notin \mathbb{D}$ in which case $v_{\mathrm{R}}(D)=\frac{1}{2}$ and $v_{\mathrm{R}}(C)=1$. Hence, $v_{\mathrm{R}}(C \wedge D) \neq 1$ and so $v_{\mathrm{R}}(\neg(C \wedge D)) \in \mathbb{D}$.

ii. Suppose $\neg(C \wedge D) \ominus$ is on $\mathrm{R}$. Then, since the tree is finished, either (1) $\neg C \ominus$ and $\neg D \ominus$ are on R or (2) all of $C \oplus$ and $\neg C \oplus$ and $D \oplus$ and $\neg D \oplus$ are on R. So, by IH, we have that either (1) $v_{\mathrm{R}}(\neg C) \notin \mathbb{D}$ and $v_{\mathrm{R}}(\neg D) \notin \mathbb{D}$ in which case $v_{\mathrm{R}}(C)=1$ and $v_{\mathrm{R}}(D)=1$ or $(2) v_{\mathrm{R}}(C) \in \mathbb{D}$ and $v_{\mathrm{R}}(\neg C) \in \mathbb{D}$ and $v_{\mathrm{R}}(D) \in \mathbb{D}$ and $v_{\mathrm{R}}(\neg D) \in \mathbb{D}$ in which case $v_{\mathrm{R}}(C)=\frac{1}{2}$ and $v_{\mathrm{R}}(D)=\frac{1}{2}$. Hence, $v_{\mathrm{R}}(C \wedge D)=1$ and so $v_{\mathrm{R}}(\neg(C \wedge D)) \notin \mathbb{D}$.

(d) Suppose $B$ is a formula of the form $C \vee D$.

i. Suppose $\neg(C \vee D) \oplus$ is on $\mathrm{R}$. Then, since the tree is finished, both $\neg C \oplus$ and $\neg D \oplus$ are on R. So, $v_{\mathrm{R}}(\neg C) \in \mathbb{D}$ and $v_{\mathrm{R}}(\neg D) \in \mathbb{D}$ by $\mathrm{IH}$, and so $v_{\mathrm{R}}(C) \neq 1$ and $v_{\mathrm{R}}(D) \neq 1$. Hence, $v_{\mathrm{R}}(C \vee D) \neq 1$ and so $\neg(C \vee D) \in \mathbb{D}$.

ii. Suppose $\neg(C \vee D) \ominus$ is on $\mathrm{R}$. Then, since the tree is finished, either $\neg C \ominus$ or $\neg D \ominus$ is on R. So, either $v_{\mathrm{R}}(\neg C) \notin \mathbb{D}$ or $v_{\mathrm{R}}(\neg D) \notin \mathbb{D}$ by $\mathrm{IH}$, and so either $v_{\mathrm{R}}(C)=1$ or $v_{\mathrm{R}}(D)=1$. Hence, $v_{\mathrm{R}}(C \vee D)=1$ and so $\neg(C \vee D) \notin \mathbb{D}$. 
(e) Suppose $B$ is a formula of the form $C \rightarrow D$.

i. Suppose $\neg(C \rightarrow D) \oplus$ is on R. Then, since the tree is finished, both $C \oplus$ and $D \ominus$ are on R. So, both $v_{\mathrm{R}}(C) \in \mathbb{D}$ and $v_{\mathrm{R}}(D) \notin$ $\mathbb{D}$ by $\mathrm{IH}$, hence $v_{\mathrm{R}}(C \rightarrow D)=0$ and $v_{\mathrm{R}}(\neg(C \rightarrow D)) \in \mathbb{D}$.

ii. Suppose $\neg(C \rightarrow D) \ominus$ is on $\mathrm{R}$. Then, since the tree is finished, either $C \ominus$ is on $\mathrm{R}$ or $D \oplus$ is on R. So, either $v_{\mathrm{R}}(C) \notin \mathbb{D}$ or $v_{\mathrm{R}}(D) \in \mathbb{D}$ by $\mathrm{IH}$, hence $v_{\mathrm{R}}(C \rightarrow D)=1$ and $v_{\mathrm{R}}(\neg(C \rightarrow$ $D)) \notin \mathbb{D}$.

(III) Second inductive case: $A$ is a formula of the form $B \wedge C$.

(a) Suppose $(B \wedge C) \oplus$ is on $\mathrm{R}$. Then, since the tree is finished, both $B \oplus$ and $C \oplus$ are on R. So, both $v_{\mathrm{R}}(B) \in \mathbb{D}$ and $v_{\mathrm{R}}(C) \in \mathbb{D}$ by IH, hence $v_{\mathrm{R}}(B \wedge C) \in \mathbb{D}$.

(b) Suppose $(B \wedge C) \ominus$ is on $\mathrm{R}$. Then, since the tree is finished, either $B \ominus$ is on $\mathrm{R}$ or $C \ominus$ is on R. So, either $v_{\mathrm{R}}(B) \notin \mathbb{D}$ or $v_{\mathrm{R}}(C) \notin \mathbb{D}$ by $\mathrm{IH}$, hence $v_{\mathrm{R}}(B \wedge C) \notin \mathbb{D}$.

(IV) Third inductive case: $A$ is a formula of the form $B \vee C$.

(a) Suppose $(B \vee C) \oplus$ is on $\mathrm{R}$. Then, since the tree is finished, either $B \oplus$ is on $\mathrm{R}$ or $C \oplus$ is on R. So, either $v_{\mathrm{R}}(B) \in \mathbb{D}$ or $v_{\mathrm{R}}(C) \in \mathbb{D}$ by $\mathrm{IH}$, hence $v_{\mathrm{R}}(B \vee C) \in \mathbb{D}$.

(b) Suppose $(B \vee C) \ominus$ is on $\mathrm{R}$. Then, since the tree is finished, both $B \ominus$ and $C \ominus$ are on R. So, both $v_{\mathrm{R}}(B) \notin \mathbb{D}$ and $v_{\mathrm{R}}(C) \notin \mathbb{D}$ by $\mathrm{IH}$, hence $v_{\mathrm{R}}(B \vee C) \notin \mathbb{D}$.

(V) Fourth inductive case: $A$ is a formula of the form $B \rightarrow C$.

(a) Suppose $(B \rightarrow C) \oplus$ is on $\mathrm{R}$. Then, since the tree is finished, either $B \ominus$ is on $\mathrm{R}$ or $C \oplus$ is on R. So, either $v_{\mathrm{R}}(B) \notin \mathbb{D}$ or $v_{\mathrm{R}}(C) \in \mathbb{D}$ by $\mathrm{IH}$, hence $v_{\mathrm{R}}(B \rightarrow C) \in \mathbb{D}$.

(b) Suppose $(B \rightarrow C) \ominus$ is on $\mathrm{R}$. Then, since the tree is finished, both $B \oplus$ and $C \ominus$ are on R. So, both $v_{\mathrm{R}}(B) \in \mathbb{D}$ and $v_{\mathrm{R}}(C) \notin \mathbb{D}$ by IH, hence $v_{\mathrm{R}}(B \rightarrow C) \notin \mathbb{D}$.

Theorem 3.3.2 (Completeness of TNH). If $X \vDash_{\mathrm{MH}} A$, then $X \vdash_{\mathrm{TMH}} A$. 
Proof. Let $X \nvdash_{\mathrm{TMH}} A$. Fix any finite $X^{\prime} \subseteq X$. Then there is an open TNH tree from $X^{\prime}$ to $C$ by definition of provability in TNH. Fix any open branch $\mathrm{R}$ of this tree with its induced interpretation $v_{\mathrm{R}}$. Since the initial list is the root of every branch, we have $B \oplus$ on $\mathrm{R}$ for all $B \in X^{\prime}$ and we have $A \ominus$ on R. It follows by Lemma 3.3.1 that $v_{\mathrm{R}}(B) \in \mathbb{D}$ for all $B \in X$ and $v_{\mathrm{R}}(A) \notin \mathbb{D}$, hence $X^{\prime} \not \nvdash_{\mathrm{MH}} A$. Generalizing, there is no finite $X^{\prime} \subseteq X$ such that $X^{\prime} \vDash_{\mathrm{MH}} C$. Thus, $X \nvdash_{\mathrm{MH}} A$ by the compactness of $\mathrm{NH}$.

Theorem 3.3.3 (Soundness of TNH). If $X \vdash_{\mathrm{TMH}} A$, then $X \vDash_{\mathrm{MH}} A$.

Proof Sketch. The soundness proof, again, makes use of a small lemma. For any branch $\mathrm{R}$ of a TNH tree, define a faithful interpretation to be an NH valuation $v$ that assigns $v(A) \in \mathbb{D}$ if $A \oplus$ is on $\mathrm{R}$ and $v(A) \notin \mathbb{D}$ if $A \ominus$ is on $\mathrm{R}$. It is straightforward to show that if $v$ is faithful to an initial segment of any branch, then when an expansion rule is applied, $v$ is faithful to at least one extension of that segment, and therefore to one branch with that segment. We infer soundness as follows. Suppose that $X \nvdash_{\mathrm{NH}} A$. Then there is an NH valuation $v$ such that $v(B) \in \mathbb{D}$ for all $B \in X$ but $v(A) \notin \mathbb{D}$. Fix any finite $X^{\prime} \subseteq X$ and any finished TNH tree from $X^{\prime}$ to $A$. By definition $v$ is faithful to the root of this tree, so it is faithful to at least one branch $\mathrm{R}$ of the tree. If $\mathrm{R}$ were a closed branch, it would either have $A \oplus$ and $A \ominus$ on it for some formula $A$, or it would have $A \ominus$ and $\neg A \ominus$ on it for some formula $A$, both of which are impossible. Generalizing, there is no finite $X^{\prime} \subseteq X$ such that all TNH trees from $X^{\prime}$ to $A$ are closed. Therefore, $X \nvdash_{\mathrm{TNH}} A$.

This demonstrates the adequacy of TNH with respect to NH. Although tableaux systems may not be considered the most natural representation of reasoning, they do have the advantage of serving as a decision procedure for our logics.

\section{Conclusion}

This incursion into unfamiliar territory has shown a new way to look at paracomplete and paraconsistent views on negation and rationality. What is the behavior of incompleteness, i.e. how ought it to affect our reasoning when we introduce contradictories both of which may be rejected? What is the behavior of consistency, i.e. how ought it to affect our reasoning when we introduce contradictories which may not both be accepted? The two logics we have explored here offer novel and interesting ways to get to grips with 
such phenomena. 9

\section{Appendix}

\section{Meta-inferences that hold in both $\mathrm{HMH}$ and $\mathrm{HNH}$}

Since the following proofs apply to both Hilbert systems, we drop subscripts on the turnstile here.

\section{The Deduction Theorem: $X, A \vdash C \Longleftrightarrow X \vdash A \rightarrow C$}

The RLD simply follows from MP. For the LRD, suppose that $X, A \vdash C$. Recall that a derivation of $X, A \vdash C$ is a sequence of formulas $A_{1}, \ldots, A_{n}$ such that $A_{n}=C$ and every $A_{i}$ in the sequence is either a member of $X \cup\{A\}$, an axiom, or follows from previous formulas in the sequence by MP. We prove by induction on $i=1,2, \ldots$ that $X \vdash A \rightarrow A_{i}$, and so $X \vdash A \rightarrow C$ in particular.

(I) Base case: for $i=1$ either $A_{1} \in X \cup\{A\}$ or $A_{1}$ is an axiom.

(a) If $A_{1} \in X \cup\{A\}$, we have either $A_{1}=A$ or $A_{1} \neq A$.

i. If $A_{1}=A$, then since $\emptyset \vdash A \rightarrow A$ (see the Iden theorem below), we have $X \vdash A \rightarrow A$, i.e. $X \vdash A \rightarrow A_{1}$.

ii. If $A_{1} \neq A$, then since $A_{1} \vdash A \rightarrow A_{1}$ from Ax.1 and MP and since $A_{1} \in X$, we have $X \vdash A \rightarrow A_{1}$.

(b) If $A_{1}$ is an axiom, we have $\emptyset \vdash A \rightarrow A_{1}$ from Ax.1 and MP, and so $X \vdash A \rightarrow A_{1}$.

(II) Inductive case: for $i>1$ there are three possibilities, two of which are similar to the above. The third possibility is that there are $A_{j}$ and $A_{k}=\left(A_{j} \rightarrow A_{i}\right)$ earlier in the sequence from which $A_{i}$ follows by MP. We have the following induction hypothesis for $1 \leq h<i$.

$$
\text { (IH) } X \vdash A \rightarrow A_{h}
$$

In particular, we have $X \vdash A \rightarrow A_{j}$ and $X \vdash A \rightarrow\left(A_{j} \rightarrow A_{i}\right)$. We can then chain these derivations together and extend them by the following steps.

$$
\text { - } A \rightarrow A_{j}
$$

\footnotetext{
${ }^{9}$ This work originated from discussions with my fellow postgrads, Doug Owings and Aaron Cotnoir, and my then-supervisor, Jc Beall, while I was still completing my Ph.D. at the University of Connecticut many years ago. Owings (2012), in particular, presented some ideas about non-classical logic that were then entirely new to me and spurred my thoughts in directions that ultimately culminated in this paper. Any errors or omissions in the final product, however, are entirely my responsibility.
}

Australasian Journal of Logic (14:1) 2017, Article no. 11 
- $A \rightarrow\left(A_{j} \rightarrow A_{i}\right)$

:

- $\left(A \rightarrow\left(A_{j} \rightarrow A_{i}\right)\right) \rightarrow\left(\left(A \rightarrow A_{j}\right) \rightarrow\left(A \rightarrow A_{i}\right)\right) \quad$ Ax.2

- $\left(A \rightarrow A_{j}\right) \rightarrow\left(A \rightarrow A_{i}\right) \quad$ MP

- $A \rightarrow A_{i}$

MP

RBC (Reasoning by Cases): $X, A \vdash C$ and $X, B \vdash C \Longleftrightarrow X, A \vee B \vdash C$

(I) For the LRD, let $X, A \vdash C$ and $X, B \vdash C$. Then, by the deduction theorem, we have $X \vdash A \rightarrow C$ and $X \vdash B \rightarrow C$ and so, by Ax.8 and MP, we have $X \vdash(A \vee B) \rightarrow C$. Thus, again by the deduction theorem, we have $X, A \vee B \vdash C$.

(II) For the RLD, let $X, A \vee B \vdash C$. Then, by the deduction theorem, we have $X \vdash(A \vee B) \rightarrow C$. It follows easily by Ax.6 and Tran that $X \vdash A \rightarrow C$ and so, again by the deduction theorem, we have $X, A \vdash C$. It follows similarly by Ax.7, etc., that $X, B \vdash C$.

\section{Theorems and Derived Rules in both $\mathrm{HMH}$ and $\mathrm{HNH}$}

Iden: $A \rightarrow A$

(1) $A \rightarrow((A \rightarrow A) \rightarrow A)$

Ax.1

(2) $(A \rightarrow(A \rightarrow A)) \rightarrow(A \rightarrow A)$

Ax.2, 1, MP

(3) $A \rightarrow(A \rightarrow A)$

Ax.1

(4) $A \rightarrow A$

$2,3, \mathrm{MP}$

Adj: $A, B \Longrightarrow A \wedge B$

(1) $A$

ASS.

(2) $B$

ASS.

(3) $A \rightarrow(B \rightarrow A)$

Ax.1

(4) $B \rightarrow A$

$1,3, \mathrm{MP}$

(5) $(B \rightarrow A) \rightarrow((B \rightarrow B) \rightarrow(B \rightarrow(A \wedge B)))$

Ax.5

(6) $(B \rightarrow B) \rightarrow(B \rightarrow(A \wedge B))$

4, 5, MP

(7) $B \rightarrow B$

Iden 

(8) $B \rightarrow(A \wedge B)$
6, 7, MP
(9) $A \wedge B$
$2,8, \mathrm{MP}$

Tran: $A \rightarrow B, B \rightarrow C \Longrightarrow A \rightarrow C$
(1) $A \rightarrow B$
(2) $B \rightarrow C$
(3) $A \rightarrow(B \rightarrow C)$
(4) $(A \rightarrow B) \rightarrow(A \rightarrow C)$
(5) $A \rightarrow C$

ASS.

ASS.

Ax.1, 2, MP

Ax.2, 3, MP

1, 4, MP

Perm: $A \rightarrow(B \rightarrow C) \Longrightarrow B \rightarrow(A \rightarrow C)$
(1) $A \rightarrow(B \rightarrow C)$
(2) $(A \rightarrow B) \rightarrow(A \rightarrow C)$
(3) $((A \rightarrow B) \rightarrow(A \rightarrow C)) \rightarrow(B \rightarrow(A \rightarrow B) \rightarrow(A \rightarrow C))$
(4) $B \rightarrow((A \rightarrow B) \rightarrow(A \rightarrow C))$
(5) $(B \rightarrow(A \rightarrow B)) \rightarrow(B \rightarrow(A \rightarrow C))$
(6) $B \rightarrow(A \rightarrow B)$
(7) $B \rightarrow(A \rightarrow C)$

ASS. Ax.2, 1, MP

Ax.1

2, 3, MP

Ax.2, 4, MP

Ax.1

$5,6, \mathrm{MP}$

\section{Theorems and Derived Rules only in $\mathrm{HMH}$}

IFN: in one direction, viz. $\nabla A \rightarrow \nabla \neg A$
(1) $\neg A \rightarrow(A \vee \neg A)$
Ax.7
(2) $\neg \neg A \rightarrow(A \vee \neg A)$
Ax.9, Ax.6, Tran
(3) $(\neg A \vee \neg \neg A) \rightarrow(A \vee \neg A)$
Ax.8, 1, 2, MP
(4) $(\neg A \vee \neg \neg A) \vee \neg(\neg A \vee \neg \neg A)$
Ax.10, Def. $\nabla$
(5) $((\neg A \vee \neg \neg A) \rightarrow(A \vee \neg A)) \rightarrow(\neg(A \vee \neg A) \rightarrow \neg(\neg A \vee \neg \neg A))$
(6) $\neg(A \vee \neg A) \rightarrow \neg(\neg A \vee \neg \neg A)$
Ax.12, 4, MP
$3,5, \mathrm{MP}$

IFN: in the other direction, viz. $\nabla \neg A \rightarrow \nabla A$

Australasian Journal of Logic (14:1) 2017, Article no. 11 
(1) $A \rightarrow(\neg A \vee \neg \neg A)$

Ax.9, Ax.7, Tran

(2) $\neg A \rightarrow(\neg A \vee \neg \neg A)$

Ax.6

(3) $(A \vee \neg A) \rightarrow(\neg A \vee \neg \neg A)$

Ax.8, 1, 2, MP

(4) $(A \vee \neg A) \vee \neg(A \vee \neg A)$

Ax.10

(5) $((A \vee \neg A) \rightarrow(\neg A \vee \neg \neg A)) \rightarrow(\neg(\neg A \vee \neg \neg A) \rightarrow \neg(A \vee \neg A))$

Ax.12, 4, MP

(6) $\neg(\neg A \vee \neg \neg A) \rightarrow \neg(A \vee \neg A)$

$3,5, \mathrm{MP}$

MEX: $\neg A \rightarrow(A \rightarrow B)$ and $\nabla A \rightarrow(\neg A \rightarrow B)$

(1) $\neg A \rightarrow(\neg A \vee \nabla A)$

Ax.6

(2) $(\neg A \vee \nabla A) \rightarrow(A \rightarrow B)$

Ax.15

(3) $\neg A \rightarrow(A \rightarrow B)$

1, 2, Tran

(4) $\nabla A \rightarrow \nabla \neg A$

IFN

(5) $\nabla \neg A \rightarrow(\neg \neg A \vee \nabla \neg A)$

Ax.7

(6) $(\neg \neg A \vee \nabla \neg A) \rightarrow(\neg A \rightarrow B)$

Ax.15

(7) $\nabla A \rightarrow(\neg A \rightarrow B)$

4, 5, 6, Tran

MRed: $A \rightarrow(\neg A \vee \nabla A) \Longrightarrow \neg A \vee \nabla A$

(1) $A \rightarrow(\neg A \vee \nabla A)$

ASS.

(2) $\neg A \rightarrow(\neg A \vee \nabla A)$

Ax.6

(3) $\nabla A \rightarrow(\neg A \vee \nabla A)$

Ax.7

(4) $((A \vee \neg A) \vee \nabla A) \rightarrow(\neg A \vee \nabla A)$

Ax.8, 1, 2, 3, MP

(5) $(A \vee \neg A) \vee \nabla A$

Ax.10

(6) $\neg A \vee \nabla A$

4, 5, MP

IncC: $\nabla(A \wedge B) \Longrightarrow(A \vee \nabla A)$ and $(B \vee \nabla B)$
(1) $\nabla(A \wedge B)$
(2) $\neg A \rightarrow(\neg A \vee \neg B)$
(3) $\neg B \rightarrow(\neg A \vee \neg B)$
(4) $\neg(A \wedge B) \rightarrow((\neg \neg A \vee \nabla \neg A) \wedge(\neg \neg B \vee \nabla \neg B))$
(5) $(\neg A \vee \neg B) \rightarrow((\neg \neg A \vee \nabla \neg A) \wedge(\neg \neg B \vee \nabla \neg B))$
(6) $\neg A \rightarrow(\neg \neg A \vee \nabla \neg A)$

ASS.

Ax.6

Ax.7

MEX, 1, MP

Ax.13, 4, Tran

2, 5, Ax.4, Tran

Australasian Journal of Logic (14:1) 2017, Article no. 11 

(7) $\neg \neg A \vee \nabla \neg A$
(8) $\neg \neg A \rightarrow(A \vee \nabla A)$
(9) $\nabla \neg A \rightarrow(A \vee \nabla A)$
(10) $A \vee \nabla A$
(11) $\neg B \rightarrow(\neg \neg B \vee \nabla \neg B)$
(12) $\neg \neg B \vee \nabla \neg B$
(13) $\neg \neg B \rightarrow(B \vee \nabla B)$
(14) $\nabla \neg B \rightarrow(B \vee \nabla B)$
(15) $B \vee \nabla B$

IncD: $\nabla(A \vee B) \Longrightarrow(\neg A \vee \nabla A)$ and $(\neg B \vee \nabla B)$
6 , Red Ax.9, Ax.6, Tran IFN, Ax.7, Tran Ax.8, 7, 8, 9, MP 3, 5, Ax.4, Tran 11, Red Ax.9, Ax.6, Tran IFN, Ax.7, Tran Ax.8, 12, 13, 14, MP
(1) $\nabla(A \vee B)$
(2) $A \rightarrow(A \vee B)$
(3) $B \rightarrow(A \vee B)$
(4) $(A \vee B) \rightarrow((\neg A \vee \nabla A) \wedge(\neg B \vee \nabla B))$
(5) $A \rightarrow(\neg A \vee \nabla A)$
(6) $B \rightarrow(\neg B \vee \nabla B)$
(7) $\neg A \vee \nabla A$
(8) $\neg B \vee \nabla B$

ASS.

Ax.6

Ax.7

MEX, 1, MP

2, 4, Ax.2, Tran

3,4 , Ax.2, Tran

5 , Red

6 , Red

\section{Theorems and Derived Rules only in $\mathrm{HNH}$}

CFN: in one direction, viz. $\bigcirc A \rightarrow O \neg A$
(1) $(\neg A \wedge \neg \neg A) \rightarrow A$
Ax.4, Ax.9, Tran
(2) $(\neg A \wedge \neg \neg A) \rightarrow \neg A$
Ax.3
(3) $(\neg A \wedge \neg \neg A) \rightarrow(A \wedge \neg A)$
Ax.5, 1, 2, MP
(4) $\circ(A \wedge \neg A)$
Ax.17
(5) $((\neg A \wedge \neg \neg A) \rightarrow(A \wedge \neg A)) \rightarrow(\neg(A \wedge \neg A) \rightarrow \neg(\neg A \wedge \neg \neg A))$
Ax.19, 4, MP
(6) $\neg(A \wedge \neg A) \rightarrow \neg(\neg A \wedge \neg \neg A)$
$1,15, \mathrm{MP}$

CFN: in the other direction, viz. $\bigcirc \neg A \rightarrow \mathrm{O} A$ 
(1) $(A \wedge \neg A) \rightarrow \neg A$

Ax. 4

(2) $(A \wedge \neg A) \rightarrow \neg \neg A$

Ax.3, Ax.9, Tran

(3) $(A \wedge \neg A) \rightarrow(\neg A \wedge \neg \neg A)$

Ax.5, 1, 2, MP

(4) $\bigcirc(\neg A \wedge \neg \neg A)$

Ax.17

(5) $((A \wedge \neg A) \rightarrow(\neg A \wedge \neg \neg A)) \rightarrow(\neg(\neg A \wedge \neg \neg A) \rightarrow \neg(A \wedge \neg A))$

Ax.19, 4, MP

(6) $\neg(\neg A \wedge \neg \neg A) \rightarrow \neg(A \wedge \neg A)$

1, 15, MP

NEX: $\bigcirc A \rightarrow((A \wedge \neg A) \rightarrow B)$

(1) $\circ(A \wedge \neg A)$

Ax.17

(2) $\mathrm{O} A \rightarrow \mathrm{O}(A \wedge \neg A)$

Ax.1, 1, MP

(3) $\circ A \rightarrow \neg(A \wedge \neg A)$

Ax.9, Def.o

(4) $\circ A \rightarrow(\circ(A \wedge \neg A) \wedge \neg(A \wedge \neg A))$

Ax.5, 2, 3, MP

(5) $(\mathrm{O}(A \wedge \neg A) \wedge \neg(A \wedge \neg A)) \rightarrow((A \wedge \neg A) \rightarrow B)$

Ax.22

(6) $\circ A \rightarrow((A \wedge \neg A) \rightarrow B)$

4, 5, Tran

LEM: $A \vee \neg A$
(1) $\neg((A \wedge \neg A) \wedge \mathrm{O} A)$
(2) $\bigcirc A \vee \neg \bigcirc A$
(3) $\circ A \rightarrow(\neg A \vee \neg \neg A)$
(4) $\neg A \rightarrow(A \vee \neg A)$
(5) $\neg \neg A \rightarrow(A \vee \neg A)$
(6) $(\neg A \vee \neg \neg A) \rightarrow(A \vee \neg A)$
(7) $\circ A \rightarrow(A \vee \neg A)$

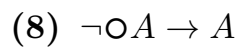
(9) $\neg \mathrm{O} A \rightarrow(A \vee \neg A)$
(10) $(\mathrm{O} A \vee \neg \mathrm{O} A) \rightarrow(A \vee \neg A)$
(11) $A \vee \neg A$

Ax.17, Def.o Ax.6, Ax.21, 1, MP Ax.6, Ax.21, Tran

Ax.7

Ax.9, Ax.6, Tran Ax.8, 4, 5, MP

3,6 , Tran Ax.9, Ax.3, Tran Ax.6, 8, Tran Ax. 8, 7, 9, MP 2, 10, MP

Dial: $\neg \mathrm{O} A \rightarrow A$ and $\neg \mathrm{O} A \rightarrow \neg A$

(1) $\neg \mathrm{O} A \rightarrow(A \wedge \neg A)$

Ax.9

(2) $(A \wedge \neg A) \rightarrow A$

Ax.3

Australasian Journal of Logic (14:1) 2017, Article no. 11 

(3) $\neg \mathrm{O} A \rightarrow A$
(4) $(A \wedge \neg A) \rightarrow \neg A$
(5) $\neg \mathrm{O} A \rightarrow \neg A$

1, 2, Tran

Ax.4

1,4 , Tran

NCon: $\neg A \rightarrow(\mathrm{O} A \rightarrow \neg(A \wedge B))$

(1) $\neg A \rightarrow(\mathrm{O} A \rightarrow \neg A)$

Ax.1

(2) $(A \wedge B) \rightarrow A$

Ax.3

(3) $\circ A \rightarrow((A \wedge B) \rightarrow A)$

Ax.1, 2, MP

(4) $\circ A \rightarrow(((A \wedge B) \rightarrow A) \rightarrow(\neg A \rightarrow \neg(A \wedge B)))$

Ax.19

(5) $(\circ A \rightarrow((A \wedge B) \rightarrow A)) \rightarrow(\circ A \rightarrow(\neg A \rightarrow \neg(A \wedge B)))$

Ax.2, 4, MP

(6) $\circ A \rightarrow(\neg A \rightarrow \neg(A \wedge B))$

$3,5, \mathrm{MP}$

(7) $(\circ A \rightarrow \neg A) \rightarrow(\circ A \rightarrow \neg(A \wedge B))$

Ax.2, 6, MP

(8) $\neg A \rightarrow(\mathrm{O} A \rightarrow \neg(A \wedge B))$

1,7 , Tran

NRed: $A \rightarrow \neg A \Longrightarrow \neg A$
(1) $A \rightarrow \neg A$
(2) $\neg A \rightarrow \neg A$
(3) $(A \vee \neg A) \rightarrow \neg A$
(4) $\neg A$

ASS.

Iden

Ax.8, 1, 2, MP

LEM, 3, MP

ConC: positive case, viz. $\bigcirc(A \wedge B) \Longrightarrow(A \wedge B) \rightarrow(\circ A \vee \neg O B)$
(1) $\circ(A \wedge B)$
ASS.
(2) $\bigcirc \neg(A \wedge B)$
CFN, 1, MP
(3) $\neg \mathrm{O} A \rightarrow \neg A$
Dial
(4) $\neg A \rightarrow(\neg(A \wedge B) \vee((A \wedge \neg A) \wedge(B \wedge \neg B)))$
Ax.6, Ax.21, Tran
(5) $\neg(A \wedge B) \rightarrow(\circ B \rightarrow \neg(A \wedge B))$
Ax.1
(6) $((A \wedge \neg A) \wedge(B \wedge \neg B)) \rightarrow \neg B$
Ax.4, Ax.4, Tran
(7) $\neg B \rightarrow(\mathrm{o} B \rightarrow \neg(A \wedge B))$
NCon
(8) $((A \wedge \neg A) \wedge(B \wedge \neg B)) \rightarrow(\circ B \rightarrow \neg(A \wedge B))$
6,7 , Tran
(9) $(\neg(A \wedge B) \vee((A \wedge \neg A) \wedge(B \wedge \neg B))) \rightarrow(\circ B \rightarrow \neg(A \wedge B))$

(10) $\neg \mathrm{O} A \rightarrow(\mathrm{O} B \rightarrow \neg(A \wedge B))$

Ax.8, 5, 8, MP $3,4,9$, Tran 
(11) $(\neg \circ A \wedge \circ B) \rightarrow(\circ B \rightarrow \neg(A \wedge B))$

Ax.3, 10, Tran

(12) $((\neg \circ A \wedge \circ B) \rightarrow \circ B) \rightarrow((\neg \circ A \wedge \circ B) \rightarrow \neg(A \wedge B))$

Ax.2, 11, MP

(13) $(\neg \circ A \wedge \circ B) \rightarrow \neg(A \wedge B)$

Ax.4, 12, MP

(14) $\neg \neg(A \wedge B) \rightarrow \neg(\neg \mathrm{O} A \wedge \mathrm{O} B)$

Ax.19, 2, 13, MP

(15) $(A \wedge B) \rightarrow(\mathrm{O} A \vee \neg \mathrm{O} B)$

Ax.9, Ax.6, Ax.21, 14, Tran

ConC: negative case, viz. $\bigcirc(A \wedge B) \Longrightarrow \neg(A \wedge B) \rightarrow((\neg A \wedge \circ A) \vee(\neg B \wedge \circ B))$

(1) $\circ(A \wedge B)$

ASS.

(2) $(\neg(\neg A \wedge \mathrm{O} A) \wedge \neg(\neg B \wedge \mathrm{O} B)) \rightarrow \neg(\neg A \wedge \mathrm{O} A)$

Ax.3

(3) $\neg(\neg A \wedge \mathrm{O} A) \rightarrow(\neg \neg A \vee \neg \mathrm{O} A)$

Ax.6, Ax.21, Tran

(4) $\neg \neg A \rightarrow A$

Ax.9

(5) $\neg \circ A \rightarrow A$

Dial

(6) $(\neg \neg A \vee \neg \mathrm{O} A) \rightarrow A$

Ax.8, 4, 5, MP

(7) $(\neg(\neg A \wedge \circ A) \wedge \neg(\neg B \wedge \circ B)) \rightarrow A$

$2,3,6$, Tran

(8) $(\neg(\neg A \wedge \circ A) \wedge \neg(\neg B \wedge \circ B)) \rightarrow \neg(\neg B \wedge \circ B)$

Ax.4

(9) $\neg(\neg B \wedge \circ B) \rightarrow(\neg \neg B \vee \neg O B)$

Ax.6, Ax.21, Tran

(10) $\neg \neg B \rightarrow B$

Ax.9

(11) $\neg \mathrm{O} B \rightarrow B$

Dial

(12) $(\neg \neg B \vee \neg \mathrm{O} B) \rightarrow B$

Ax.8, 10, 11, MP

(13) $(\neg(\neg A \wedge \circ A) \wedge \neg(\neg B \wedge \circ B)) \rightarrow B$

$8,9,12$, Tran

(14) $(\neg(\neg A \wedge \circ A) \wedge \neg(\neg B \wedge \circ B)) \rightarrow(A \wedge B)$

Ax.5, 6, 13, MP

(15) $\neg(A \wedge B) \rightarrow \neg(\neg(\neg A \wedge \circ A) \wedge \neg(\neg B \wedge \circ B))$

Ax.19, 1, 14, MP

(16) $\neg(\neg(\neg A \wedge \mathrm{O} A) \wedge \neg(\neg B \wedge \mathrm{O} B)) \rightarrow(\neg \neg(\neg A \wedge \mathrm{O} A) \vee \neg \neg(\neg B \wedge \mathrm{O} B))$

Ax.6, Ax.21, Tran

(17) $(\neg \neg(\neg A \wedge \circ A) \vee \neg \neg(\neg B \wedge \mathrm{\circ} B)) \rightarrow((\neg A \wedge \circ A) \vee(\neg B \wedge \circ B))$

Ax.9, Ax.6, Ax.7, Ax.8, MP

(18) $\neg(A \wedge B) \rightarrow((\neg A \wedge \circ A) \vee(\neg B \wedge \circ B))$

15, 16, 17, Tran

ConD: positive case, viz. $\bigcirc(A \vee B) \Longrightarrow(A \vee B) \rightarrow((A \wedge \circ A) \vee(B \wedge \circ B))$

(1) $\bigcirc(A \vee B)$

ASS.

(2) $\bigcirc \neg(A \vee B)$

CFN, 1, MP

(3) $(\neg(A \wedge \mathrm{O} A) \wedge \neg(B \wedge \mathrm{O} B)) \rightarrow \neg(A \wedge \mathrm{O} A)$

Ax.3

Australasian Journal of Logic (14:1) 2017, Article no. 11 

(4) $\neg(A \wedge \mathrm{O} A) \rightarrow(\neg A \vee \neg \mathrm{O} A)$
Ax.6, Ax.21, Tran
(5) $\neg A \rightarrow \neg A$
Iden
(6) $\neg \mathrm{O} A \rightarrow \neg A$
Dial
(7) $(\neg A \vee \neg \mathrm{O} A) \rightarrow \neg A$
Ax.8, 5, 6, MP
(8) $(\neg(A \wedge \circ A) \wedge \neg(B \wedge \circ B)) \rightarrow \neg A$
$3,4,7$, Tran
(9) $(\neg(A \wedge \circ A) \wedge \neg(B \wedge \circ B)) \rightarrow \neg(B \wedge \circ B)$
Ax. 4
(10) $(\neg(B \wedge \mathrm{O} B) \rightarrow(\neg B \vee \neg O B)$
Ax.6, Ax.21, Tran
(11) $\neg B \rightarrow \neg B$
Iden
(12) $\neg \mathrm{O} B \rightarrow \neg B$
Dial
(13) $(\neg B \vee \neg O B) \rightarrow \neg B$
Ax.8, 11, 12, MP
(14) $(\neg(A \wedge \circ A) \wedge \neg(B \wedge \circ B)) \rightarrow \neg B$
9, 10, 13, Tran
(15) $(\neg(A \wedge \mathrm{O} A) \wedge \neg(B \wedge \mathrm{O} B)) \rightarrow(\neg A \wedge \neg B)$
Ax.5, 8, 14, MP
(16) $(\neg(A \wedge \circ A) \wedge \neg(B \wedge \circ B)) \rightarrow \neg(A \vee B)$
Ax.20, 15, Tran
(17) $\neg \neg(A \vee B) \rightarrow \neg(\neg(A \wedge \circ A) \wedge \neg(B \wedge \circ B))$
Ax.19, 2, 16, MP
(18) $(A \vee B) \rightarrow \neg(\neg(A \wedge \circ A) \wedge \neg(B \wedge \circ B))$
Ax.9, 17, Tran
(19) $\neg(\neg(A \wedge \circ A) \wedge \neg(B \wedge \circ B)) \rightarrow(\neg \neg(A \wedge \circ A) \vee \neg \neg(B \wedge \circ B))$
Ax.6, Ax.21, Tran
(20) $(\neg \neg(A \wedge \mathrm{O} A) \vee \neg \neg(B \wedge \mathrm{O} B)) \rightarrow((A \wedge \mathrm{O} A) \vee(B \wedge \mathrm{O} B))$
Ax.9, Ax.6, Ax.7, Ax.8, MP
(21) $(A \vee B) \rightarrow((A \wedge \circ A) \vee(B \wedge \circ B))$
18, 19, 20, Tran

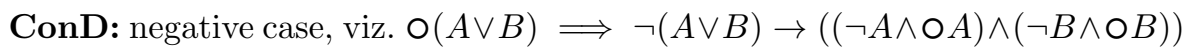

(1) $\circ(A \vee B)$

ASS.

(2) $\neg(\neg A \wedge \mathrm{O} A) \rightarrow(\neg \neg A \vee \neg \mathrm{O} A)$

Ax.6, Ax.21, Tran

(3) $\neg \neg A \rightarrow(A \vee B)$

Ax.9, Ax.6, Tran

(4) $\neg \mathrm{O} A \rightarrow(A \vee B)$

(5) $(\neg \neg A \vee \neg \mathrm{O} A) \rightarrow(A \vee B)$

Dial, Ax.6, Tran

(6) $\neg(\neg A \wedge \circ A) \rightarrow(A \vee B)$

Ax.8, 3, 4, MP

2,5 , Tran

(7) $\neg(\neg B \wedge \circ B) \rightarrow(\neg \neg B \vee \neg \circ B)$

Ax.6, Ax.21, Tran

(8) $\neg \neg B \rightarrow(A \vee B)$

Ax.9, Ax.7, Tran

(9) $\neg \mathrm{O} B \rightarrow(A \vee B)$

Dial, Ax.7, Tran

(10) $(\neg \neg B \vee \neg O B) \rightarrow(A \vee B)$

Ax. 8, 8, 9, MP

(11) $\neg(\neg B \wedge \mathrm{O} B) \rightarrow(A \vee B)$

7, 10, Tran

Australasian Journal of Logic (14:1) 2017, Article no. 11 
(12) $(\neg(\neg A \wedge \mathrm{O} A) \vee \neg(\neg B \wedge \mathrm{O} B)) \rightarrow(A \vee B)$

Ax.8, 6, 11, MP

(13) $\neg(A \vee B) \rightarrow \neg(\neg(\neg A \wedge \circ A) \vee \neg(\neg B \wedge \circ B))$

Ax.19, 1, 12, MP

(14) $\neg(\neg(\neg A \wedge \circ A) \vee \neg(\neg B \wedge \circ B)) \rightarrow(\neg \neg(\neg A \wedge \circ A) \wedge \neg \neg(\neg B \wedge \circ B))$

Ax.20

(15) $(\neg \neg(\neg A \wedge \circ A) \wedge \neg \neg(\neg B \wedge \circ B)) \rightarrow((\neg A \wedge \circ A) \wedge(\neg B \wedge \circ B))$

Ax.3, Ax.4, Ax.9, Ax.5, MP

(16) $\neg(A \vee B) \rightarrow((\neg A \wedge \circ A) \wedge(\neg B \wedge \circ B))$

13, 14, 15, Tran

Australasian Journal of Logic (14:1) 2017, Article no. 11 


\section{References}

Beall, J. (2015). Free of detachment: Logic, rationality, and gluts. Nô̂s, $49(2): 410-423$.

Beziau, J.-Y. and Franceschetto, A. (2015). Strong three-valued paraconsistent logics. In Beziau, J.-Y., Chakraborty, M., and Dutta, S., editors, New Directions in Paraconsistent Logic, pages 131-145. Springer.

da Costa, N. C. (1974). On the theory of inconsistent formal systems. Notre Dame Journal of Formal Logic, 15(4):497-510.

Dummett, M. (1959). Truth. Proceedings of the Aristotelian Society, 59(1):141-62.

Field, H. (2008). Saving Truth From Paradox. Oxford University Press.

Goldberg, H., Leblanc, H., and Weaver, G. (1974). A strong completeness theorem for 3-valued logic. Notre Dame Journal of Formal Logic, $15(2): 325-330$.

Kleene, S. C. (1952). Introduction to Metamathematics. North Holland.

Kripke, S. (1975). Outline of a theory of truth. Journal of Philosophy, 72(19):690-716.

Marcos, J. (2005). On a problem of da Costa. In Sica, G., editor, Essays on the Foundations of Mathematics and Logic, volume 2, pages 53-69. Polimetrica.

Owings, D. C. (2012). Indeterminacy and Logical Atoms. PhD thesis, University of Connecticut.

Priest, G. (1979). The logic of paradox. Journal of Philosophical Logic, $8(1): 219-241$.

Priest, G. (2006). In Contradiction: A Study of the Transconsistent. Oxford University Press, 2nd edition.

Sette, A. (1973). On the propositional calculus $p^{1}$. Mathematica Japonicae, 18(13):173-180.

Sette, A. and Carnielli, W. A. (1995). Maximal weakly-intuitionistic logics. Studia Logica, 55(1):181-203.

Williamson, T. (1994). Vagueness. Routledge.

Australasian Journal of Logic (14:1) 2017, Article no. 11 